\title{
Chapter Abstract
}

Nowadays the realization of market-competitive devices based on nanomaterials is a major challenge. Optimization of the device performance requires deep understanding of the physical phenomena at the nanoscale. In this context, electron beambased techniques become indispensable. Several instruments have been developed in the past decades to provide versatile diagnostic solutions for improving materials, designs and device fabrication. These characterization techniques applied to nanostructured semiconductors can help filling the gap between material science and engineering by bringing in light important physical parameters.

In this Chapter, the family of electron beam-based techniques is briefly introduced. First, the electron beam/matter interaction is described both in physical and operational terms. In particular, different phenomena occurring when a flux of electron collides with a semiconductor material are discussed. Then, two main electron beam scanning techniques are discussed in the following sections: electron beam induced current microscopy and cathodoluminescence. After a short description of the fundamentals, for each technique a bibliographic review is presented to illustrate its applications to analyses of semiconductor nanowires.

Keywords: Electron Beam, Scanning Techniques, Electron beam induced current, Cathodoluminescence, Semiconductor nanowires 


\title{
Characterisation of Semiconductor Nanowires by Electron Beam Induced Microscopy and Cathodoluminescence
}

\author{
Valerio Piazza ${ }^{1}$, Gwénolé Jacopin ${ }^{2}$, Maria Tchernycheva ${ }^{1}$ \\ ${ }^{1}$ Centre de Nanosciences et de Nanotechnologies, UMR 9001 CNRS, Université Paris Sud, Université \\ Paris-Saclay, 10 Boulevard Thomas Gobert, 91120 Palaiseau, France \\ ${ }^{2}$ Univ. Grenoble Alpes, CNRS, Grenoble INP, Institut Néel, 38000 Grenoble, France \\ Corresponding Author Contact: maria.tchernycheva@u-psud.fr
}

The use of scanning beam probe microscopy techniques is fundamental in the development of novel devices. Indeed, thanks to the advances in the epitaxial growth and nanofabrication, almost all electronic and optical components are based on materials having a sub-micron size in one or several dimensions. The optimization of the device performance requires a careful design and control of the active semiconductor materials. The assessment of the properties of nanometric sized materials is still an open task. For this reason, a comprehensive characterization at the nanoscale is necessary to determine their advantages and drawbacks for different applications.

Among the characterization techniques suited for this purpose, electron beambased techniques have gained a lot of interest in the last years due to the strong and highly localized interaction of the electron beam with the matter. By coupling the high resolution with the typical scanning mode of the electronic microscopes, it is possible to analyze large surfaces with high accuracy and in a short time. Beside the classical structural and compositional characterization methods (such as scanning electron microscopy SEM, transmission electron microscopy TEM, scanning transmission electron microscopy STEM, Energy-dispersive X-ray spectroscopy EDS, etc), other types of electron beam-based techniques have been developed specifically for the investigation of semiconductor materials. Due to the physical phenomena occurring upon electron exposure, the electron beam is able to excite excess carriers in semiconductors, which can probe the electrical and the optical properties of the sample. For this reason, dedicated techniques and setups have been developed, aiming to access the electronic properties of semiconductor materials. These techniques already give interesting insights for the analysis of bulk and layered materials, but they become indispensable for the investigation of nanostructures. Beside the analysis of the material properties, electron beam-based techniques have been largely used to assess the physical phenomena in a wide variety of optoelectronic devices such as light emitting diodes (LEDs), solar cells and photodetectors. These techniques are particularly useful for the investigation of nanostructured devices (e.g. nanowire-based LEDs and solar cells that will be discussed in this chapter). 
This Chapter starts with a brief Section which describes the electron beam/semiconductor interaction, defining the spatial resolution and carrier injection and focusing on the variation of these quantities by tuning the electron beam excitation. This introduction provides understanding of the basic operation principles of two electron probe techniques discussed in this Chapter, namely the electron beam induced current microscopy (EBIC) and the cathodoluminescence (CL). Both EBIC and CL have gained the interest of the semiconductor nanomaterial community in the last years due to their versatility and the large amount of information that they can provide about the investigated specimen. These techniques will be described in this Chapter by giving the working principles and the main technical aspects. In each Section, a large bibliographic review is reported to give an overview of the different applications of these techniques for the characterization of different types of nanowires currently under investigation for the development of novel optoelectronic devices.

\section{Electron beam/Matter Interaction}

When a solid material is exposed to a high-energy electron beam $(3 \mathrm{kV}-30 \mathrm{kV})$, a high number of interactions occurs due to elastic and inelastic scattering of the injected electrons. The scattering events lead to a transfer of energy from the beam to the material which can induce a large number of phenomena, such as emission of secondary electrons (SE), backscattered electrons (BSE), Auger electrons (AE) and $\mathrm{X}$-rays. The peculiarity of each interaction and the ability to detect the emitted particles is generally used to develop characterization techniques which allow to get access to specific material properties. Classical examples are given by the detection of secondary electrons, which is used to perform morphological analysis (SE images), and by the analysis of the X-ray emissions, used for compositional analysis of the investigated material (XEDS).

In semiconductor materials, the inelastic scattering of the injected electrons induces the generation of $\mathrm{e}^{-/} \mathrm{h}^{+}$pairs, analogously to the photon excitation. Thanks to this phenomenon and to the small volume affected by the beam exposure, the electron beam techniques offer the possibility to investigate locally the properties related to the electronic bands of the material. The main differences with respect to the case of the excitation of carriers by light exposure depend on characteristic features of the electron beam/samples interaction, i.e. on the electron scattering and on the carrier generation.

\section{(a) Electron scattering and interaction volume}

Due to the high energy of the injected electrons, multiple scattering events occur in the material and therefore, this process affects a certain region. This region is called interaction volume and it is the volume of semiconductor material where the generation of excess carriers occurs under electron beam exposure. The interaction volume can extend from few tens of nanometers up to few hundreds of microns 
depending on the nature of the investigated materials and on the operational conditions. As it is intuitive, the interaction volume is intimately related to the spatial resolution achievable by a scanning probe technique. For this reason, the interaction volume is a key element to be taken in consideration for all the scanning beam techniques and its peculiarities must be carefully evaluated.

The spatial resolution is defined by the shape and size of the interaction volume, which, being related to the electron scattering, depends on the primary electron energy and on the material (chemical composition, density, scattering cross-section). Pear, spherical and hemispherical shapes are obtained for increasing atomic numbers ${ }^{1,2}$. Although it concerns few electron probe techniques, it is to be noticed that the shape of the interaction volume affects also the sensitivity to the surface properties, since the volume involved as a function of the penetration depth can strongly change in the different cases. For instance, this is important for the morphological analysis by SEM microscopy where the emission of a large ratio of secondary electrons from the surface is desirable.

Concerning the techniques which probe the bulk properties of the material, the importance of the shape is related to the ratio between the penetration depth of the beam into the material and the lateral size of the interaction volume which strongly affects the spatial resolution of the scanning techniques. These parameters can be tuned by varying the energy of the injected electron, i.e. the acceleration voltage of the beam $\left(\mathrm{V}_{\mathrm{acc}}\right)$. Indeed, the energy of the injected electrons increases with the acceleration voltage. As a consequence, in bulk materials the number of sequential scattering events per injected electrons increases, resulting in a deeper penetration of the interaction volume. For this reason, high voltage beams are generally used to probe structures or insertion buried into the material ${ }^{3,4}$. However, contextually to the penetration depth, the lateral size of the interaction volume increases, leading to a reduction of the spatial resolution. Depending on the systems under investigation, a thorough optimization could be required to achieve a fine resolution when investigating buried elements.

To estimate the interaction volume in a material with a given geometry and for given experimental conditions, Monte Carlo simulations can be used. This stochastic simulation forecasts the electron scattering phenomena by calculating the trajectories considering random scattering events in a stepwise manner. The energy lost by the injected electrons can be evaluated with this method as well. The loss of energy is a fundamental parameter since the electrons need a threshold energy value to be able to induce the physical phenomenon used to probe the sample (such as Xrays emission, secondary electron emission). For this reason, the boundaries of the interaction volume cannot be considered abrupt. Depending on the physical phenomenon used to probe the properties of the material, the dimensions of the interaction volume have to be properly defined. In the case of the generation of $\mathrm{e}^{-} / \mathrm{h}^{+}$ pairs in semiconductor materials, several models have been developed to define this threshold value, called cut-off energy. The cut-off energy, in fact, affects the dimensions of the interaction volume, when estimating the resolution of a scanning probe techniques on a given sample. This is illustrated in Figure 1a, which shows an example of a Monte Carlo simulation of the electron energy loss in a GaAs layer for a beam with acceleration voltage of $10 \mathrm{kV}$. The lines represent the iso-energetic 
distribution of the scattered electrons and the numbers indicate the percentage of energy lost outside the given iso-energy line. As a general rule in bulk materials, the interaction volume increases with increasing beam acceleration voltage.

Another factor that strongly affects the spatial resolution of the electron probe techniques is the carrier diffusion length in the material ${ }^{5}$, which can be influenced by bandgap gradients, charge barriers, heterostructures or passivated surfaces. For the sake of simplicity, let's consider the case of an optical emission upon carrier recombination. Indeed, if the carrier diffusion length is much larger than the lateral size of the interaction volume, the generated carriers can travel over large distances before recombining. In this case, the recombination occurs further from the injection point and outside the interaction volume, and therefore the spatial resolution is not related to its dimensions. In other terms, the spatial resolution is limited by the carrier diffusion length in the material. This is typically the case of III-V thin films or Si structures (independently on its geometry), which are characterized by diffusion length from $1 \mu \mathrm{m}$ to $100 \mu \mathrm{m}^{6,7}$. On the contrary, if the carrier diffusion length is comparable with the lateral size of the interaction volume, the charges excited in the interaction volume poorly diffuse before recombining and the spatial resolution is mostly given by the geometry of the interaction volume. This is mostly the case of III-V NWs which are characterized by low values of diffusion length (50 - 150 $\mathrm{nm})^{8,9}$

When probing nanostructured materials, it has to be taken into account that the penetration depth can be as large as, or even larger than the thickness of the investigated sample. In this case, the shape and size of the interaction volume is strongly modified due to the partial transmission of the beam and the presence of backscattered electrons (BSEs) from the substrate, if there is any (Figure 1b). The transmission of the electrons causes a narrowing of the interaction volume when increasing the acceleration voltage (contrary to the bulk case) and therefore an increase of the resolution, similarly to the case of transmission scanning techniques (such as TEM, STEM $)^{10}$. However, if a substrate is present on the beam path, the effect of BSEs, which lead to an enlargement of the interaction volume, can be dominant and no improvement in resolution is obtained.

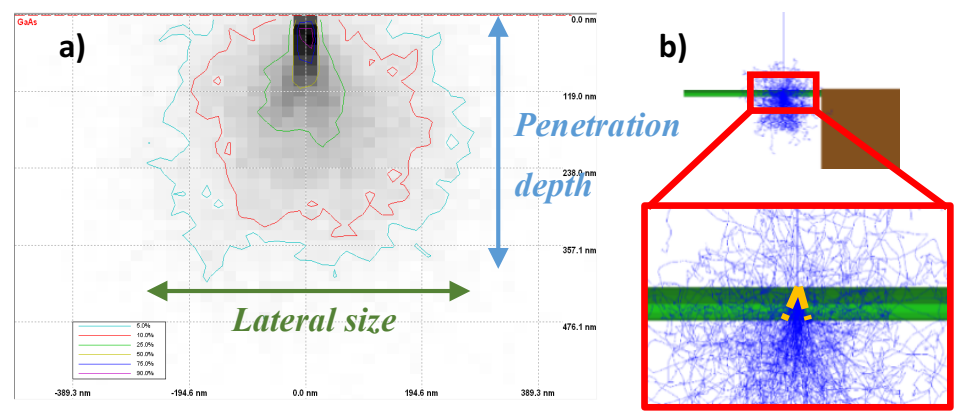

Figure 1 a) Example of a Monte Carlo simulation and definition of the penetration depth and of the lateral size of the interaction volume considering a 5\% cut-off. Different cut-off energies are shown by the colored lines representing the iso-energetic distribution of the 
scattered electrons. b) Simulation of the electron trajectory after the interaction with a suspended GaAs NW. In the red-framed inset, the partial transmission of the electron beam can be observed.

(b) Carrier generation: theoretical models and operational aspects

The generation of excess carriers in the interaction volume is fundamental for probing the properties of the investigated samples. Different models were proposed to describe the carrier generation in semiconductor materials under an electron beam irradiation. The main works were produced by Donolato ${ }^{11-13}$, who developed a model assuming that, at a fixed depth, the ionization density follows a radial Gaussian distribution. Under this assumption, it is possible to describe the local carrier generation induced by the electron beam as a function $g(z, r ; R)$, where $\mathrm{z}, \mathrm{r}$ represent the geometrical variables (respectively, the depth and the radius) and $\mathrm{R}=\mathrm{R}(\mathrm{E})$ is the electron energy distribution (as bi-dimensionally illustrated in Figure 1). In other terms, this function allows to determine the number of carriers generated per unit time in a certain point within the interaction volume, depending on the energy of the electrons scattered in this point. The local generation function can be expressed as:

$$
g(z, r ; R)=\frac{A(z, R)}{2 \pi \sigma^{2}(z, R)} \exp \left[-\frac{r^{2}}{2 \sigma^{2}(z, R)}\right],
$$

where $A$ and $\sigma$ represent the amplitude and the width of the ionization density. The great advantage of this model is that the properties of the material which affect the carrier generation are fully embedded in two independent functions, i.e. the ionization density approximated with a Gaussian function and the electron energy distribution $\mathrm{R}(\mathrm{E})$. Providing these functions, the proposed model gives a universal mathematical description of the number of carriers generated per unit time by an electron beam injected in a semiconductor material.

On the other hand, the mentioned functions which take into account the nature of the material (in particular the ionization density) have to be considered case by case since they depend on both material properties and electron beam parameters. For this reason, the model has been further developed in following works ${ }^{14,15}$. In particular, Leamy described the local carrier generation with a function which splits the geometrical and the intensive factors, expressed in the form:

$$
g(z, r)=\langle g\rangle \frac{V_{a c c} I_{b}(1-f)}{q E_{e h}},
$$

where $\langle g\rangle$ is the normalized energy distribution of the electrons within the interaction volume, $V_{a c c}$ and $I_{b}$ are the main beam parameters, i.e. the acceleration voltage and the beam current impinging the surface, $f$ is the energy fraction of the incident electron beam that is reflected from the sample surface, $q$ is the electron charge and $E_{e h}$ is the energy fraction spent by the electrons to excite the electron-hole pairs, which is a constant for a given material and independent on the electron beam. To be noticed that the model from Leamy requires the same information as in Eq. 1: the electron energy distribution and the ionization energy. The latter is here considered through the factor $E_{\text {eh }}$, which is roughly equal to three times the material band 
gap energy $\left(3 \mathrm{E}_{\mathrm{g}}\right)$, if the losses due to the interaction with phonons are neglected (for more details, please refer to ref. ${ }^{16}$ ).

This simplified expression allows to determine the variation of the local generation rate with the operation parameters and thus it has a more practical application for the description of experimental cases. In fact, by integrating Eq. 2, it is possible to define the overall generation rate $\mathrm{G}_{0}$ (Eq. 3), which enables the calculation of the carrier concentration $(\Delta \text { p) under beam exposure (Eq. } 4)^{17}$ :

$$
\begin{gathered}
G_{0} \approx \frac{1}{3 q E_{g}} \frac{V_{a c c}}{V} I_{b} \\
\Delta \mathrm{p}=G_{0} \tau \approx \frac{1}{3 q E_{g}} \frac{V_{a c c}}{V} I_{b} \tau,
\end{gathered}
$$

where $V$ is the total interaction volume and $\tau$ is the carrier lifetime in the medium. Equation 1.4 indicates that the excess carrier density in the interaction volume changes linearly with the beam current, i.e. the flux of injected electrons, and therefore it is possible to control the excitation in the material by tuning $\mathrm{I}_{\mathrm{b}}$. An example is shown in Figure 2, which displays the dependence of the generated carrier concentration in bulk n-doped $\mathrm{GaN}$ as a function of the beam current. The lines in the plot are calculated at a fixed acceleration voltage following the model by Leamy expressed by Eq. 2, 3 and 4. To perform the calculations, the parameter $f$ is considered as half of the backscattered coefficient for the material ${ }^{\mathrm{a}}$, as suggested in the work of Leamy ${ }^{14}$. The lifetime of both electrons and holes is assumed to be $1 \mathrm{~ns}$, in agreement with values reported in the literature for $\mathrm{GaN}$ (refs. ${ }^{18,19}$ and references therein). Moreover, the diffusion of the induced carriers out of the interaction volume is neglected for the sake of simplicity. For more details, please refer to ref. ${ }^{20}$.

Looking at this relation one could naively think that the same effect can be achieved by varying the acceleration voltage, however this isn't true. As previously discussed, the acceleration voltage affects the shape and the dimensions of the interaction volume and therefore by tuning $\mathrm{V}_{\text {acc }}$, the total interaction volume $\mathrm{V}$ is also changed. In bulk materials (or more generally, in specimen thick enough to prevent the electron beam transmission), the interaction volume scales with the acceleration voltage faster than the generation rate ${ }^{20}$. In other terms, the overall effect is that, at a fixed beam current, the generated carrier density decreases when $V_{\text {acc }}$ is increased (Figure 2).

The control of the excess carrier concentration is fundamental for the analysis of material, since the relation between the generated carrier density and the free carrier concentration can affect the transport phenomena in the materials ${ }^{21}$. If the excess carrier density is comparable or higher than the equilibrium carrier concentration (either the intrinsic concentration $-\mathrm{n}_{0}$ - or the intentional doping level in doped semiconductors $-\mathrm{N}_{\mathrm{a}}$ or $\mathrm{N}_{\mathrm{d}}$ ), the induced current is ruled by ambipolar diffu-

a The $f$ values determined for beam voltages of $2 \mathrm{keV}, 3 \mathrm{keV}, 5 \mathrm{keV}, 7 \mathrm{keV}, 10$ $\mathrm{keV}, 15 \mathrm{keV}, 20 \mathrm{keV}, 25 \mathrm{keV}$, and $30 \mathrm{keV}$ were $0.135,0.135,0.131,0.125,0.124$, $0.121,0.118,0.118$, and 0.117 , respectively. 
sion. In this regime both electrons and holes contribute to the current and the definition of minority and majority carriers becomes meaningless. This situation is referred to as high injection regime (HIR). On the contrary, if the generated carrier density is much lower than the equilibrium concentration, a so-called low injection regime (LIR) is established and the collected current is given by minority carrier diffusion. As it will be discussed in the following Sections, the latter regime is highly desirable to investigate material parameters such as the minority carrier diffusion length.

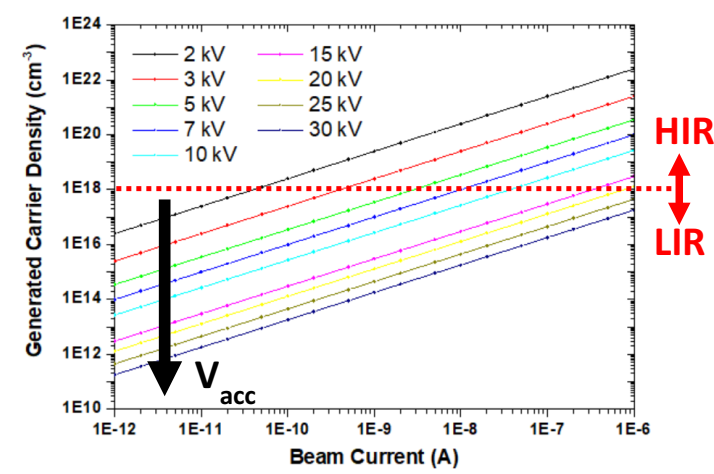

Figure 2 Generated carrier concentration in bulk n-doped GaN as a function of the beam current: the colored straight lines are calculated at a fixed acceleration voltage which is increasing as indicated by the black arrow. The horizontal dotted red line indicates the high injection regime limit, assuming a background doping of $10^{18} \mathrm{~cm}^{-3}$ : above this line the excitation occurs in a high injection regime (HIR), while below it occurs in a low injection regime (LIR).

\section{Electron Beam Induced Current Mapping}

Electron Beam Induced Current microscopy (EBIC) is a probe scanning technique which enables the investigation of internal electric fields in semiconductor materials. EBIC microscopy has been widely used in optoelectronics, and in particular solar cells, for more than thirty years ${ }^{22}$. In 2D solar cells, EBIC was used to probe the electrical activity of the devices with a high resolution, to extract material parameters and to detect failures induced by material defects. Thanks to the development of nano- and micro- fabrication of electrical contacts, more recently EBIC mapping has been applied to the investigation of the physical properties of nanostructured materials. Concerning nanowires, its use has ranged from the estimation of material parameters such as minority carrier diffusion length and active dopants concentration to the investigation of complex heterostructures embedded in the nano-objects.

\subsection{Measurement configurations}


The working principle of EBIC microscopy is based on the ability to collect in an electrical circuit the generated carriers drifted by an internal field present in the investigated specimen. The drift of excess carriers generated by the electron beam and their diffusion towards the internal field offer the possibility to extract a socalled "induced current" in the external circuit (Figure 3a). By scanning the electron beam on a line or on a surface, an EBIC profile or map can be built where each pixel indicates the induced current measured when the electron beam was injected in that position. Due to the nanometric extension of the interaction volume (as previously mentioned), EBIC allows to visualize and to analyze internal fields at the nanoscale, and therefore the electrical properties of the material can be locally investigated.

Generally, different configurations can be used to perform EBIC measurements depending on the geometry and the internal structure of the investigated material. Both factors, in fact, play a key role in the interpretation of the maps and profiles, especially in the case of individual NWs since EBIC mapping is sensitive to the direction of the internal electric fields with respect to the direction of the electron beam. For the sake of clarity, in the following the case of an EBIC scan along the axis of a NW containing a p-n junction is considered: this configuration is called cross-sectional.

If the NW contains an axial junction, the built-in field $(\vec{E})$ is oriented perpendicularly to the electron beam, i.e. the active region is confined in the scan direction. Therefore, a non-negligible induced current is recorded only in the vicinity of the junction during cross-sectional measurements and both diffusive and drift transport regimes can be analyzed ${ }^{23}$. A schematic of this configuration is shown in Figure $3 \mathrm{~b}$ where an example of current profile (i.e. the current recorded in each position along the scanned axis) is shown for the case of a NW containing an axial junction. This configuration enables also the analysis of the carrier diffusion, which manifests as an exponential variation of the induced current along the scan direction. Several models have been proposed to describe the induced current profile as a function of the minority carrier diffusion length $\left(L_{\text {diff }}\right)^{5,24,25}$, hence allowing to estimate this material parameter from EBIC measurements. Charge drift is characterized by a subexponential variation of the induced current (assuming an infinitesimally small interaction volume, induced current would be constant, as shown in Figure 3b). The localization of the region where carriers are drifted enables the estimate of the extension of the depletion region $\left(W_{d e p}\right)$ which depends on doping levels in the p-and $\mathrm{n}$ - parts of the $\mathrm{NW}^{4}$. It is worthy to notice that in the case of NWs containing a core/shell junction $\mathrm{L}_{\text {diff }}$ and $\mathrm{W}_{\text {dep }}$ can be investigated by cleaving the wire: the cleavage edge allows to obtain a junction directly exposed and perpendicular to the electron beam. The analysis of $\mathrm{L}_{\text {diff }}$ and $\mathrm{W}_{\text {dep }}$ through cross-sectional EBIC measurements can be used also for the optimization of NW devices, since they both contribute to define the charge collection volume, i.e. the active volume where the generated carriers can be collected in an external circuit. This is of great interest for NW solar cells ${ }^{23,26}$ and for NW light emitting diodes (LEDs) ${ }^{4,27}$, since it can be used as a feedback measurements to optimize the extension of the active region.

The EBIC analysis of a NW containing a radial p-n junction by scanning the central axis is more complex since the built-in field is parallel to the electron beam. In this case, the EBIC profile is not sensitive to the charge transport regimes, since 
charges move in the direction of the electron beam (in Figure $3 \mathrm{c}$ the red arrows indicating the electric field are parallel to the electron beam). In other terms, the extension of the depletion region and the charge diffusion cannot be addressed in this configuration. However, the profile along the axis can be analyzed by taking into account the exact geometry of the junction.

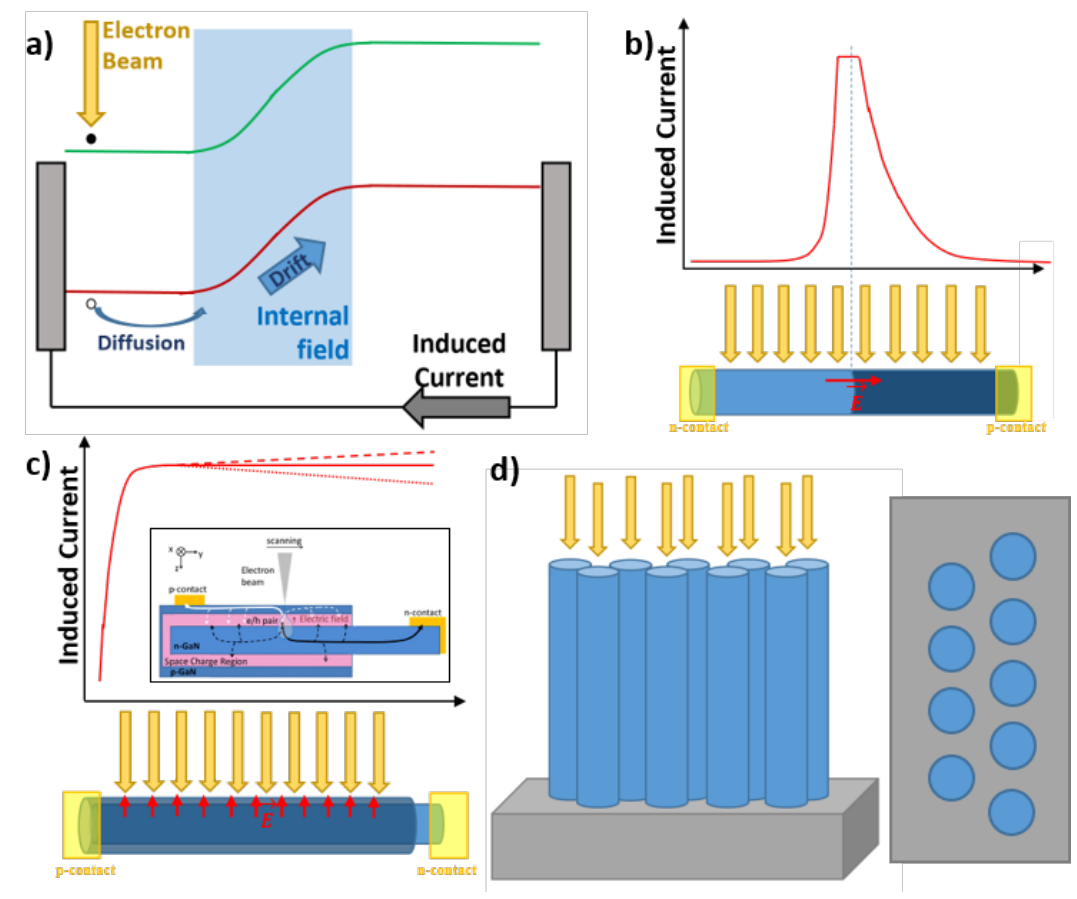

Figure 3: a) Schematic of EBIC working principle; b) Qualitative EBIC profile obtained by scanning the axis of a NW containing an axial junction; c) Qualitative EBIC profile obtained by scanning the axis of a $N W$ containing a radial junction. Solid, dashed and dotted lines represent the induced current profile depending on the ratio between core and shell resistances. In the inset, the charge collection mechanism in core/shell NWs is schematically reported. In both panels b) and c), the current profile is considered positive if it flows through the junction from the $n$-side to the p-side. d) Schematic of a top view EBIC measurement on a NW array and the corresponding top view image. In all the panels, the electron beam injection direction is indicated by yellow arrows.

[Inset in panel $\mathrm{c}$ is reproduced with permission from P Lavenus, A Messanvi, L Rigutti, A De Luna Bugallo, H Zhang,F Bayle, F H Julien, J Eymery, C Durand and M Tchernycheva, 2014, "Experimental and theoretical analysis of transport properties of core-shell wire light emitting diodes probed by electron beam induced current microscopy", Nanotech. 25(25), 255201, DOI:10.1088/0957-4484/25/25/255201. Copyright (2014) IOP Publishing]

In fact, the geometry of a real NW containing a radial junction deviates from an ideal cylinder and is not trivial: on top, the capping of the internal core typically gives rise to an axial junction (i.e. to a presence of a non-radial electric field component). Away from the extremities along the axis, the electric field is purely radial 
in the ideal case of a defect-free structure. A schematic representation is displayed in Figure 3c. Due to this geometry, the current profile is expected to be non-linear on top of the NW and to become constant if a homogenous $\vec{E}$ is present along the length of the wire ${ }^{28}$. Therefore, in this configuration EBIC mapping can be used to assess the variation of the built-in field within the nano-object (intra-wire homogeneity). Several phenomena can lead to an inhomogeneous field, such as $1 \mathrm{D}$ defects $^{29}$, inhomogeneous doping concentration or alloy composition along the length of the wire ${ }^{30}$.

In addition to the built-in field, also the local carrier collection properties can influence the EBIC profile in this configuration. Being differently doped, core and shell conduct different type of majority carriers to the external circuit (in the inset of Figure $3 \mathrm{c}$ illustrating a core/shell nitride nanowire LED $^{31}$, holes are transported in the shell and electrons are transported in the core). It means that the resistances of the core and the shell play a role for the carrier collection ${ }^{31}$. If the shell is more resistive than the core, it limits the current collection and the EBIC profile decreases from top to the bottom, since the carriers collected in the bottom part of the shell have to overcome a higher resistance than the carriers collected in the top part of the core (dotted line in Figure 3c). Vice versa, if the core is more resistive than the shell, the EBIC profile increases from the top to the bottom (dashed line in Figure $3 \mathrm{c})$. Therefore, comparable core and shell resistance are necessary to maximize the current extraction. In this case, the EBIC profile is flat (solid line in Figure 3c).

Another possible configuration for the EBIC analysis of NWs is the top-view configuration, i.e. the electron beam and the NW axis are parallel (Figure 3d). Topview configuration can be interesting for analyses of radial-junction nanowires: similarly to the case of cross-sectional mapping, by cleaving the top part of the wire, top-view measurements enable the analysis of the internal electric field since the junction field is perpendicular to the electron beam, as was mentioned before. For axial-junction NWs, instead, the junction is buried deep from the surface and therefore, the lateral resolution is lower than in cross-sectional measurements. The extraction of the junction parameters is more complex since an analysis at different beam acceleration voltages (and thus at different beam penetration depth) needs to be carried out. As a consequence, the accuracy of this analysis is strongly dependent on the precision of the model used to describe the electron beam/wire interaction volume. For this reason, top-view analyses are usually used to get information on the wire-to-wire homogeneity of the electrical properties within an array: the topview visualization (schematically represented in Figure 3d) allows to compare the collection properties of several tens or hundreds of wires under the same excitation conditions $^{32}$, which is of great interest for NW devices.

To be noticed that performing EBIC measurements on arrays or on single NWs requires a different preparation of the samples and in particular different types of electrical contacts. For NW arrays, a top contact is required on a large area. Since the top contact is influencing the shape of the interaction volume and the energy of electrons penetrating into the semiconductor sample, the contact material and thickness should be adjusted to find a compromise between a low electrical resistance and a low attenuation of the electron beam. In NW array optoelectronic devices, the top contact is usually made of transparent conductive oxides (typically ITO), which 
has a good optical transparency over a large spectral range, although it strongly attenuates the electron beam ${ }^{33,34}$.

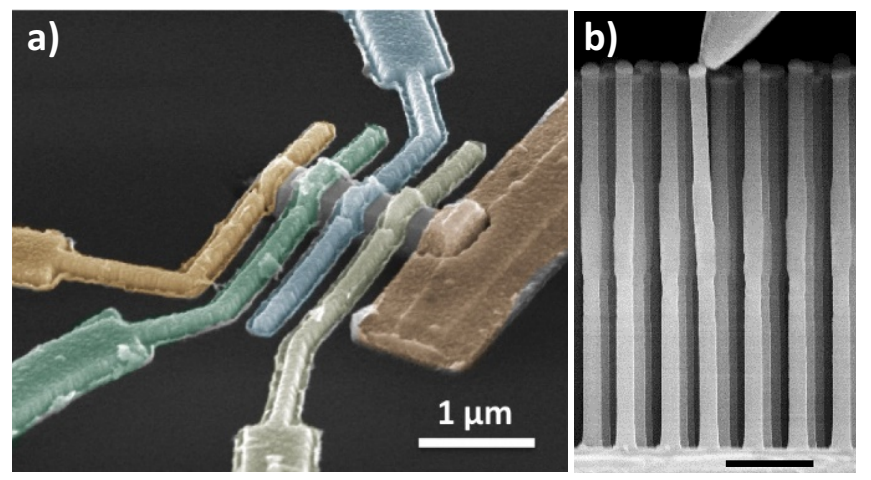

Figure 4: Examples of electrical contacts on single NWs by metal deposition (a) and by insitu nano-manipulators (b). The scale bar in panel b corresponds to $0.5 \mu \mathrm{m}$.

On the other side, the investigation of single NWs requires nanometric contacts to connect the individual nano-objects to an external electrical circuit where the induced current can be detected. This step usually involves destructive processes, since the NWs need to be detached from the substrate; in addition time- and energyconsuming fabrication steps are required to realize nano-contacts by means of electron beam lithography ${ }^{31,35}$. Although the resulting contacts are highly reliable, the contact resistance can be low and their fabrication can be very accurate (an example is given in Figure 4a), this method limits the number of NWs that can be investigated. In this context, in the last years many works have been reported in literature concerning the use of metallic nano-tips to collect electrical outputs from individual NWs, as in the case of c-AFM analysis and KPFM microscopy ${ }^{36-39}$. This is a high throughput method which allows to analyze a large number of individual NWs. The use of the nano-manipulators results in faster and more flexible measurements, since the contact points can be easily changed (Figure $4 \mathrm{~b}$ ). However, it is challenging to control the contact area and the contact resistance, which depends on the mechanical pressure applied by the tip, on the chemistry of the surface and on the work-functions of the semiconductor and of the metallic tip. This approach often results in the formation of a Schottky barrier between the tip and the NW, which can perturb the measurement.

\subsection{EBIC applied to the analysis of NW properties}

In this Section, the use of EBIC microscopy for NW analyses is discussed through a bibliographic review. As previously discussed, EBIC can be used in a versatile way to investigate several physical phenomena and material properties depending on the internal structure of the wires and the measurement configuration. 
For the sake of clarity, this Section is divided in 3 parts concerning (i) the investigation of the depletion region, (ii) the analysis of the minority carrier diffusion length and (iii) the assessment of the intra-wire and wire-to-wire homogeneity.

\section{(a) Analysis of the space charge region}

The visualization and the analysis of the space charge region (or depletion region) across a junction by EBIC mapping can provide information on the phenomena occurring in a nanostructure in its working conditions. This is particularly interesting for the development of high efficiency solar cells and LEDs where the extension of the depleted region in the active material is strongly correlated to the overall efficiency of the device.

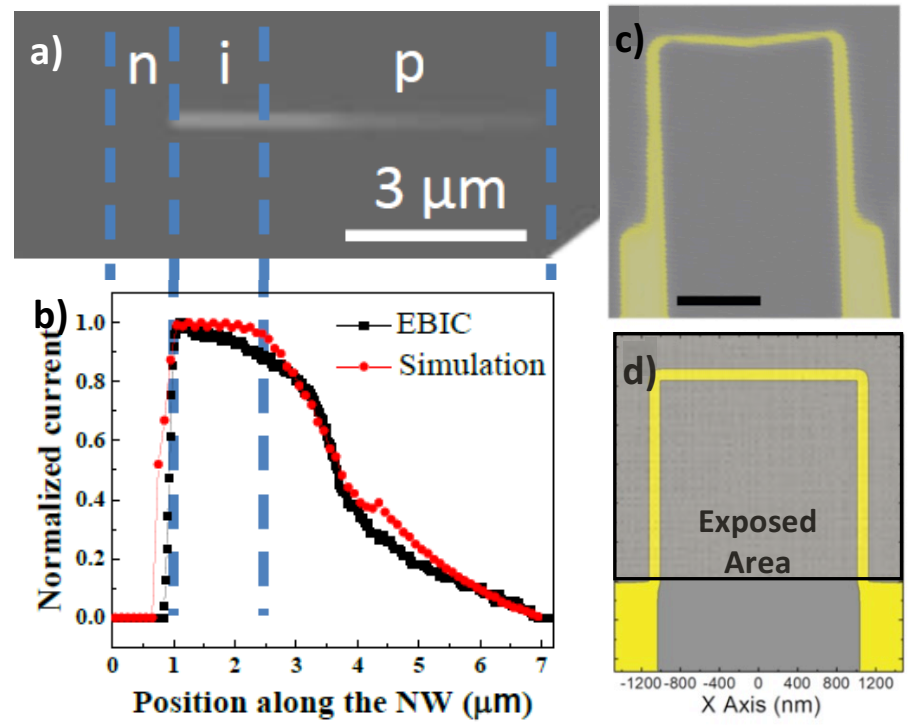

Figure 5: EBIC map (a) and profile along the longitudinal axis (b) of a single InP $N W$; c) EBIC map of a GaN/InGaN core/shell NW and (d) its schematics with respect to the beam exposure. The scale bar represents $1 \mu \mathrm{m}$.

[Panels a and b are reproduced with permission from Z. Zhong, Z. Li, Q. Gao, Z. Li, K. Peng, L. Li, S. Mokkapati, K. Vora, J. Wu, G. Zhang, Z. Wang, L. Fu, H. H. Tan, and C. Jagadish, 2016, "Efficiency Enhancement of Axial Junction InP Single Nanowire Solar Cells by Dielectric Coating", Nano energy, 28, 106-114, DOI: 10.1016/j.nanoen.2016.08.032. Copyright (2016) Elsevier. Panels c and d are reproduced and re-adapted with permission from P. Tchoulfian, F. Donatini, F. Levy, A. Dussaigne, P. Ferret, J. Pernot, 2014, "Direct imaging of p-n junction in core-shell GaN wires", Nano Lett. 14, 3491-3498, https://doi.org/10.1021/n15010493. Copyright (2014) American Chemical Society.]

An example is given by Zhong and coworkers who used EBIC mapping on InP NWs containing an axial p-i-n junction. In order to prove the effective charge collection of these structures at the nanoscale, the authors performed EBIC mapping and profiling on individual NWs, demonstrating the extraction of a high EBIC current in a large portion of the nano-object (Figure $5 \mathrm{a}$ and $\mathrm{b}$ ). Similarly, Tchoulfian et al. used EBIC to investigate the properties of GaN NWs containing a radial p-n 
junction in view of their application as light emitters ${ }^{4}$. By probing cleaved NWs, EBIC maps showed the collection of the current in correspondence of the junction position (Figure 5c), allowing to localize the field region and to estimate the doping.

In addition, EBIC maps allowed to observe dopant activation phenomena upon exposure of the inner part of the wire to the electron beam. In particular, the areas exposed to an intense electron beam showed a narrower space charge region, as schematized in Figure 5d.

To understand the relation between the depletion region extension and the doping level, it should be recalled that the depleted volume across a $p-n$ junction reduces as the doping level in the semi-junctions gets higher, due to the larger carrier density. Generally, those quantities are related as:

$$
V_{\text {dep }} \alpha \sqrt{\frac{1}{N_{d}}+\frac{1}{N_{a}}}=\sqrt{\frac{1}{N_{e f f}}},
$$

where $V_{d e p}$ is the space charge region volume, $N_{d}$ and $N_{a}$ represent respectively the donor and acceptor doping levels across the junction and $N_{\text {eff }}$ is the effective doping level. Considering a 1D model for an abrupt p-n junction, the relation between the depletion region width $(W)$ and $N_{\text {eff }}$ can be expressed as:

$$
W=\sqrt{\frac{2 \varepsilon\left(V_{b i}-V\right)}{q} \times\left(\frac{1}{N_{e f f}}\right)}
$$

where $\varepsilon$ is the permittivity of the material, $V_{b \mathrm{i}}$ is the built-in field arising across the abrupt junction, $V$ is the applied bias and $q$ is the electron charge.

Referring to the work of Tchoulfian and co-authors, they used these equations to estimate the effective doping level in the material at the nanoscale by analyzing quantitatively the EBIC maps and profiles, as shown in Figure 6a where W is experimentally estimated.

Since the model described by Eq. 5 and 6 takes also in consideration the effect of an external bias, several measurements can be performed on a single wire (Figure $6 \mathrm{~b}$ ) at different voltage. By plotting $\mathrm{W}(\mathrm{V})$ it is possible to obtain an estimation of $\mathrm{N}_{\text {eff }}$, as shown in Figure 6c. This approach has been used also to estimate separately the active acceptor and donor concentrations in a p-n junction ${ }^{40}$. This was achieved using the aforementioned EBIC analysis method in combination with voltage-induced SE contrast imaging which can be used to localize the position of an abrupt junction due to the different contrast given by $\mathrm{p}$ - and $n$ - dopants ${ }^{41,42}$. An example of this phenomenon can be observed in the SEM images of Figure $6 \mathrm{~d}$ where the secondary electron contrast allows to localize the position of the axial p-n junction. In this work, the authors investigated the possibility to obtain an Esaki diode, i.e. an abrupt heavily doped $\mathrm{p}^{++} / \mathrm{n}^{++}$junction where a tunneling current could flow under voltage $^{43,44}$. Since the depletion region width is a critical parameter for the design of an Esaki diode, EBIC mapping has been used to measure the extension of the space charge region in different wires (Figure $6 \mathrm{~d})^{45}$. The quantitative analysis of those maps by tracing the profile along the axis (reported in Figure 6e) allowed to correlate the $\mathrm{I}(\mathrm{V})$ characteristic curves of the Esaki diode with the presence of a narrow depletion region in individual nanowires. 

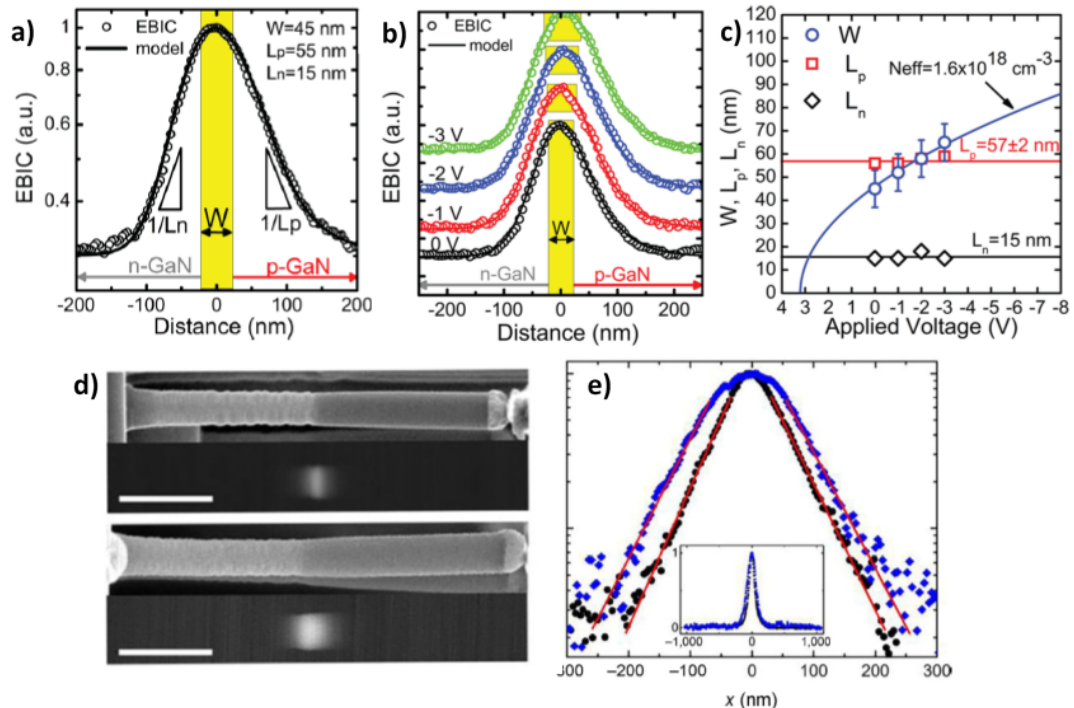

Figure 6: Estimation of the minority carrier diffusion lengths $\left(L_{n}, L_{p}\right)$ and depleted region extension (W) in an EBIC profile (a) and in several profiles acquired under several external biases (b); c) $W, L_{n}$ and $L_{p}$ as a function of the applied voltage. The plot enables the estimation of the effective doping level across the junction $\left(N_{\text {eff }}\right)$ by fitting of $W(V)$. d) SEM images and corresponding EBIC maps of two InGaP/InP NWs containing a tunnel diode; e) Axial EBIC profiles corresponding to the maps in panel d.

Panels a-c are reproduced with permission from P. Tchoulfian, F. Donatini, F. Levy, A. Dussaigne, P. Ferret, J. Pernot, 2014, "Direct imaging of $\mathrm{p}-\mathrm{n}$ junction in core-shell GaN wires", Nano Lett. 14, 34913498, DOI: 10.1021/n15010493. Copyright (2014) American Chemical Society. Panels d-e are reproduced with permission from X. Zeng, G. Otnes, M. Heurlin, R. T Mourão, and M. T. Borgström, 2018, "InP/GaInP nanowire tunnel diodes", Nano Resear., 11(5), 2523-2531, DOI: 10.1007/s12274-017-18778. Copyright (2018) Springer Nature]

\section{(b) Estimation of the minority carrier diffusion length}

While the extension of the space charge region depends on the design of the junction, the minority carrier diffusion length $\left(\mathrm{L}_{\text {diff }}\right)$ can be considered as a material parameter which measures the mean free path of the minority carriers in a doped semiconductor. Generally, $\mathrm{L}_{\text {diff }}$ can be influenced by a number of factors as defect density, impurities concentration, doping level and, in nanowires, surface-to-volume ratio ${ }^{46-48}$. For these reasons, it is a parameter of great interest for the analysis of the materials in view of their application. 

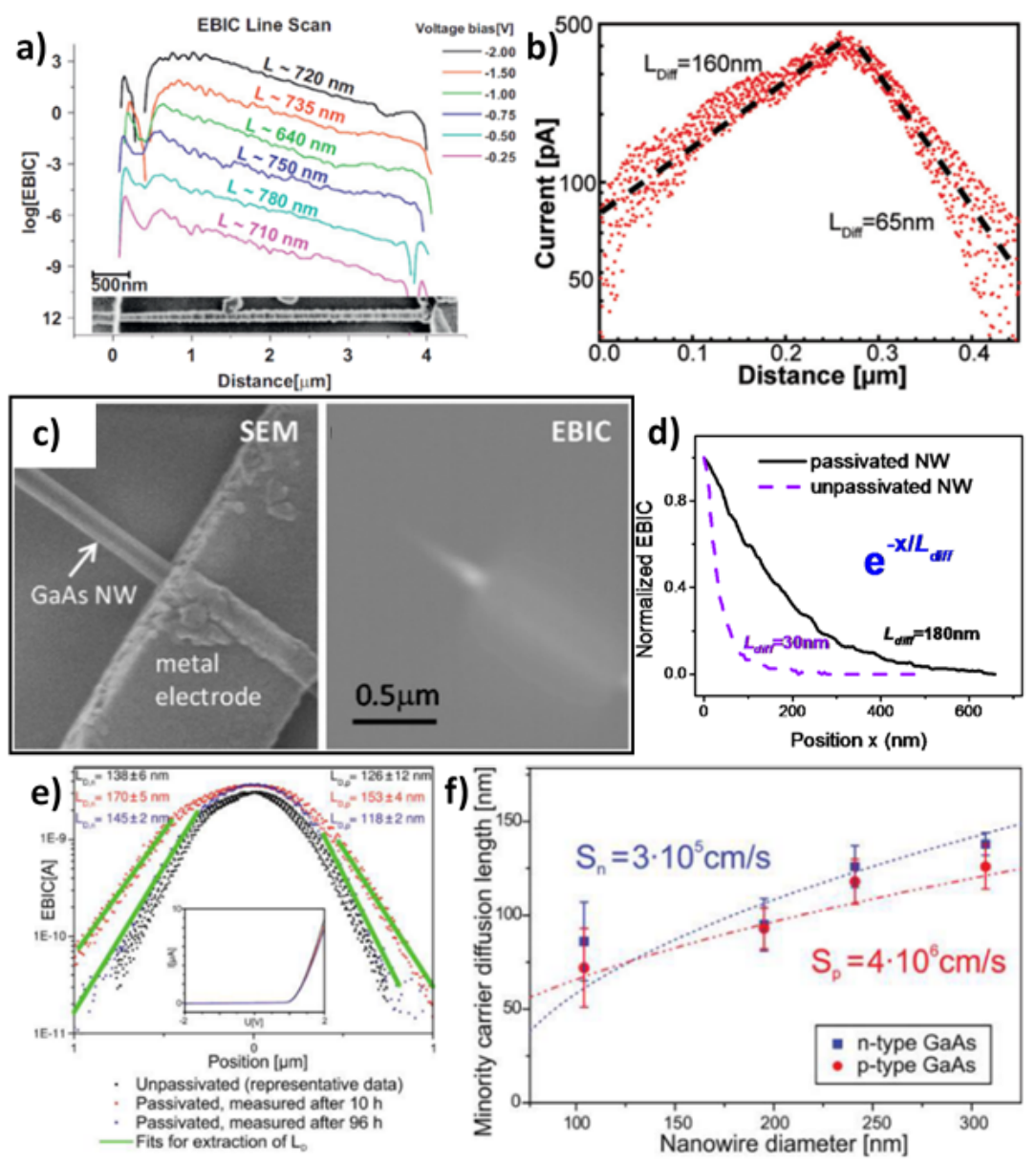

Figure 7: a) EBIC profile lines of a p-Si NW under different external bias ( $L_{\text {diff }}$ is estimated for each profile); b) EBIC profile of an InP NW containing an axial junction ( $L_{\text {diff }}$ is estimated on both p-and n-segments); c) SEM and corresponding EBIC map of a GaAs NW under a metal pad forming a Schottky contact; d) EBIC profile of the GaAs NWs with and without surface passivation; e) EBIC profiles of a GaAs NW containing a p-n junction with and without ammonium sulfide passivation; f) $L_{\text {diff }} v$ s $N W$ diameter corresponding to panel e and corresponding estimation of the surface recombination rate in p-and n-doped GaAs NWs.

[Panel a is reproduced with permission from A. Solanki, P. Gentile, V. Calvo, G. Rosaz, B. Salem, V. Aimez, D. Drouin, N. Pauc, 2012, "Geometrical control of photocurrent in active Si nanowire devices", Nano Energy 1(5), 714-722, DOI: 10.1016/j.nanoen.2012.05.010. Copyright (2012) Elsevier. Panel b is reproduced with permission from J. Wallentin, P. Wickert, M. Ek, A. Gustafsson, L. R. Wallenberg, M. H. Magnusson, L. Samuelson, K. Deppert, and M. T. Borgström, 2011, "Degenerate p-doping of InP nanowires for large area tunnel diodes", App. Phys. Lett.., 99(25), 253105, DOI: 10.1063/1.3669697. Copyright (2018) AIP Publishing. Panels $\mathrm{c}$ and d are reproduced with permission from C.-C. Chang, C.Y. Chi, M. Yao, N. Huang, C.-C. Chen, J. Theiss, A. W. Bushmaker, S. LaLumondiere, T.-W. Yeh, M. L. Povinelli, C. Zhou, P. D. Dapkus, S. Cronin, 2012, "Electrical and Optical Characterization of Surface Passivation in GaAs Nanowires", Nano Lett., 12 (9), 4484-4489, DOI: 10.1021/nl301391h. Copyright 
(2012) American Chemical Society. Panels e and f are reproduced with permission from C. Gutsche, R. Niepelt, M. Gnauck, A. Lysov, W. Prost, C. Ronning, F.-J. Tegude, 2012, "Direct Determination of Minority Carrier Diffusion Lengths at Axial GaAs Nanowire p-n Junctions", Nano Lett., 12 (3), 1453-1458, DOI: 10.1021/n1301391h. Copyright (2012) American Chemical Society.]

In order to estimate $\mathrm{L}_{\text {diff }}$ in NWs by EBIC mapping a rectifying interface is required to obtain a localized charge collection region where the carrier diffusion can be analyzed. To study homogeneous materials, a Schottky contact is intentionally induced at the interface with a metal contact for this purpose. This is the case of Solanki et al. ${ }^{49}$ who performed EBIC several line scans under different external bias on p-doped Si NWs to evaluate their suitability for photovoltaic applications (Figure 7a). An estimated average $\mathrm{L}_{\text {diff }}$ value was around $700 \mathrm{~nm}$, which is much lower than what typical values observed in high quality bulk Si $\left(\mathrm{L}_{\mathrm{diff}}>100 \mu \mathrm{m}^{6,50}\right)$. A reduction of the minority carrier diffusion length is observed in NWs with respect to the bulk material also in other materials such as $\mathrm{GaAs}, \mathrm{GaN}, \mathrm{InP}^{8,17,51}$.

On the other hand, an electrical junction is naturally present in active structures like solar cells, LEDs and transistors, and the analysis of $\mathrm{L}_{\text {diff }}$ is useful to describe both material properties and the architecture of the whole devices. For example, in the case of a NW containing an axial junction the minority carrier diffusion length is related to the ability of the structure to collect the generated carriers and therefore the $\mathrm{L}_{\text {diff }}$ value is important to determine the material quality for specific applications. For instance, high $\mathrm{L}_{\text {diff }}$ values are desirable for NW solar cells, where a large collection region needs to be achieved. On the contrary, low values are expected for an Esaki diode, where very high doping levels are necessary to maximize the tunneling properties. An example is given by Wallentin et al. who performed EBIC microscopy on InP NWs which exploited tunneling properties to prove the existence of a sharp and highly doped axial junction (Figure $7 b)^{8}$.

As previously mentioned, it has been experimentally observed that the minority carrier diffusion is lower in NWs than in bulk materials. The reason for this can be addressed to the high surface to volume ratio of these nano-objects. In this case, surface recombination rate becomes predominant and thus the minority carriers recombine faster in NWs than in bulk. The relationship between the minority carrier diffusion length and the surface in NWs has been widely investigated experimentally $^{17}$. As shown in Figure $7 \mathrm{c}$ and d, Chang et al. ${ }^{9}$ reported the EBIC analysis of $\mathrm{L}_{\text {diff }}$ in GaAs NWs with and without surface passivation, proving a 6-fold increase when saturating the surface dangling bonds.

The variation of $\mathrm{L}_{\text {diff }}$ with the surface recombination rate can be better appreciated through the equations describing the variation of the carrier lifetime in high aspect ratio structures ${ }^{52}$ (Eq. 7) and the definition of $\mathrm{L}_{\text {diff }}$ for a NW (Eq. 8):

$$
\begin{gathered}
\frac{1}{\tau_{N W}}=\frac{1}{\tau_{B}}+\frac{4 S}{d} \\
L_{\text {diff }}=\sqrt{D * \tau_{N W}}
\end{gathered}
$$

where $\tau_{\mathrm{NW}}$ and $\tau_{\mathrm{B}}$ are respectively the minority carrier lifetimes in the NW and in bulk, $\mathrm{S}$ is the surface recombination rate, $\mathrm{d}$ is the NW diameter, and $\mathrm{D}$ is the diffusion coefficient defined by the Einstein-Smoluchowski relationship. 
Gutsche et al. ${ }^{17}$ measured the $\mathrm{L}_{\text {diff }}$ of GaAs NWs containing a p-n junction with and without passivation, testing also the stability in time of the ammonium sulfide passivating action (Figure 7e). By using these equations, the surface recombination rate in p- and n-doped GaAs NWs is estimated (Figure 7f) and values comparable to the bulk materials are found. This work proved that the lower carrier lifetime and therefore lower minority carrier diffusion length is related to the large extension of the lateral surface and its recombination action. This negative effect can be alleviated by a dedicated surface passivation.

\section{(c) Homogeneity assessment by EBIC mapping}

The analysis of the space charge region and the estimation of the diffusion length can be used also to assess the homogeneity of the electrical properties in individual wires. This approach is particularly useful in the case of NWs consisting of several materials (hetero-structured NWs) or having a complex internal structure (such as wires containing quantum insertions). While the first case is typical for photovoltaic structures, the second one represents a common case for LED NWs.

A comprehensive example of intra-wire homogeneity analysis is given by the work of Tchernycheva et al. on GaN/InGaN NWs ${ }^{30}$. The SEM image of the core/shell NW and the corresponding EBIC map are shown in Figure 8a and b. In this work, the EBIC current is measured in a cross-sectional configuration upon cleavage of the NWs, in order to expose the inner ternary alloy quantum well. The authors used the current collected from the active region to describe the intra-wire variation of the electrical properties of the GaN core/shell structure. For instance, by tracing the profile at different height along the wire (Figure $8 \mathrm{c}$ ) and by estimating the $L_{\text {diff }}$ values, the homogeneity of the charge transporting layers $(n-G a N$ in the core and $\mathrm{p}-\mathrm{GaN}$ in the shell) is assessed. In fact, by overlapping the normalized radial EBIC profiles acquired at different position, it was possible to prove that a homogeneous charge transport is achieved within individual NWs, which is a necessary condition to obtain high performing nanostructured LEDs.

In addition to the radial analysis, the variation of the EBIC current along the axial direction can provide useful information on the properties of the LED. As shown in Figure 8d, a higher current is collected at the bottom of the wire and a lower current is collected at the top. Several factors could affect the axial collection properties of a core/shell wire, such as inhomogeneous properties of the core/shell junction along the axis, increased defect density on top and different core and shell resistance ${ }^{31,53}$. However, the radial analysis of the EBIC profile revealed a highly homogeneous junction while an increased defect density would have resulted in a sharper variation of the signal, as for bulk materials ${ }^{54}$. On the contrary, a higher core resistance is expected to induced a gradually decreasing EBIC signal from the bottom to the top in agreement with the observations ${ }^{31}$. 

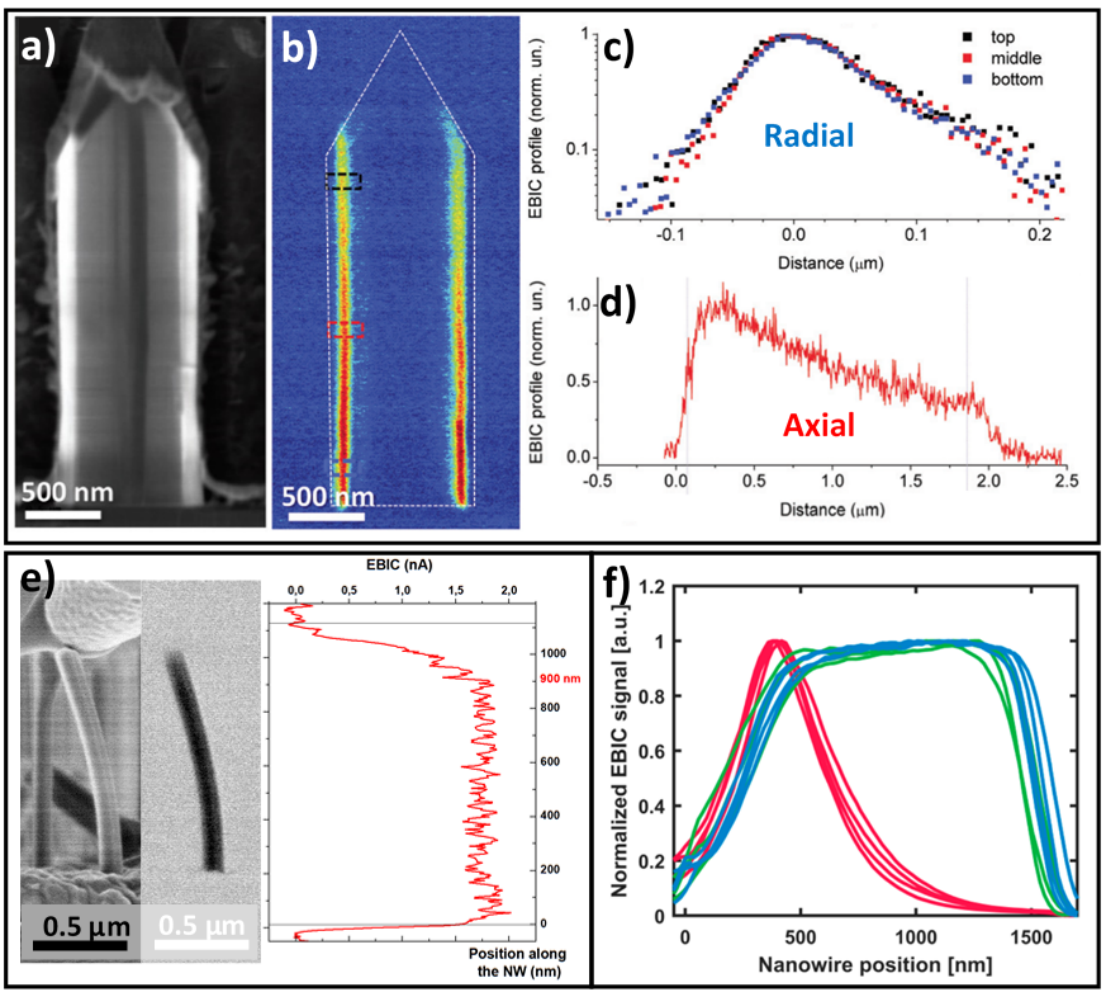

Figure 8 SEM image (a) and corresponding EBIC map (b) of a core/shell InGaN/GaN NW; c) Normalized radial EBIC profile (the origin of the horizontal axis corresponds to the maximum EBIC current value); d) Normalized axial EBIC profile (the origin of the horizontal axis corresponds to the NW/substrate interface); e) SEM image, EBIC map and corresponding axial EBIC profile of a core/shell GaAs NW; f) EBIC profiles of several InP NWs containing a nominal $p$-i-n structure with different level of compensation used in the middle segment, respectively equal to $\chi_{D E Z n}=0$ (red), $\chi_{D E Z n}=0.510^{-7}$ (green) and $\chi_{D E Z n}=2.110^{-7}$ (blue).

[Panels a-d are reproduced with permission from M. Tchernycheva, V. Neplokh, H. Zhang, P. Lavenus, L. Rigutti, F. Bayle, F. H. Julien, A. Babichev, G. Jacopin, L. Largeau, R. Ciechonski, G. Vescovi, O. Kryliouk, 2015, "Core-shell InGaN/GaN nanowire light emitting diodes analyzed by electron beam induced current microscopy and cathodoluminescence mapping", Nanoscale 7, 11692-11701, DOI: 10.1039/C5NR00623F. Copyright (2012) The Royale Society of Chemistry. Panel e is reproduced with permission from V. Piazza, M. Vettori, A. A. Ahmed, P. Lavenus, F. Bayle, N. Chauvin, F. H. Julien, P. Regreny,G. Patriarche, A. Fave, M.1 Gendry, M. Tchernycheva, 2018, "Nanoscale investigation of a radial p-n junction in self-catalyzed GaAs nanowires grown on Si (111)", Nanoscale 10, 20207-20217, DOI: 10.1039/c8nr03827a. Copyright (2012) The Royale Society of Chemistry. Panel f is reproduced with permission from G. Otnes, E. Barrigón, C. Sundvall, K. E. Svensson, M. Heurlin, G. Siefer, L. Samuelson, I. Åberg, M. T. Borgström, 2018, "Understanding InP Nanowire Array Solar Cell Performance by Nanoprobe-Enabled Single Nanowire Measurements", Nano Lett. 18, 3038-3046, DOI: 10.1021/acs.nanolett.8b00494. Copyright (2018) American Chemical Society.]

This qualitative analysis is very important for core/shell structure where the characterization of the electrical properties of the inner core is usually a challenging 
task. With this respect, EBIC microscopy can be used as a powerful characterization tool for fast analysis of NW devices. This was done by Piazza et al. ${ }^{28}$, who investigated GaAs NWs containing a radial p-n junction. By contacting a GaAs NW with a micromanipulator, as shown in the SEM image in Figure 8e, the authors observed highly constant EBIC current along the length of the wire (from both the map and the axial profile), demonstrating that the doping concentrations induce a comparable series resistance in the core and in the shell.
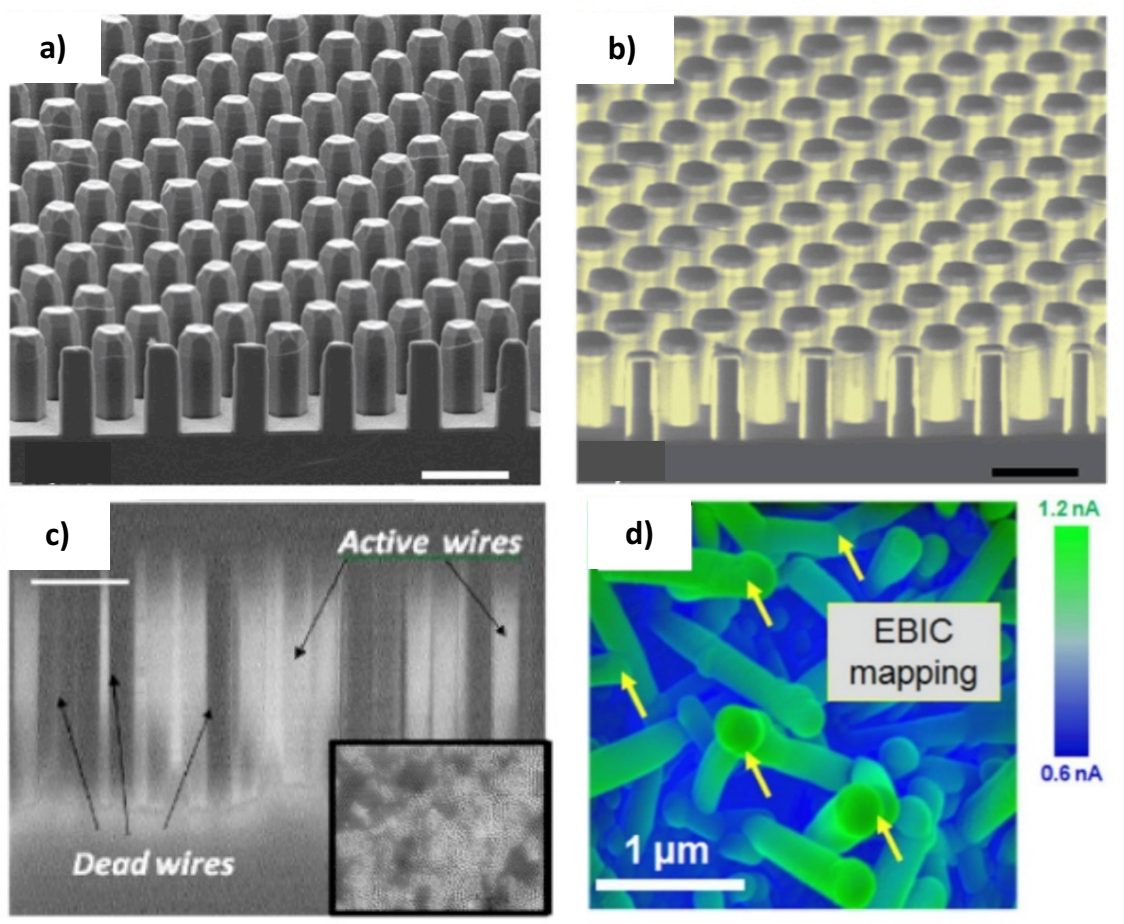

Figure 9 SEM image (a) and corresponding EBIC map (b) of a GaN/InGaN NWs array; c) Cross-section EBIC mapping of samples with "dead" and electrically active Si NWs. The scale bar represents $1 \mu \mathrm{m}$. The inset shows the EBIC top-view EBIC mapping confirming that only few nanowires are active. d) EBIC map of non-vertical Si NWs containing a radial structure.

[Panels $\mathrm{a}$ and $\mathrm{b}$ are reproduced with permission from P. Tchoulfian, F. Donatini, F. Levy, A. Dussaigne, P. Ferret, J. Pernot, 2014, "Direct imaging of p-n junction in core-shell GaN wires", Nano Lett. 14, 3491-3498, DOI: 10.1021/n15010493. Copyright (2014) American Chemical Society. Panel c is reproduced with permission from A. S. Togonal, M. Foldyna, W. Chen, J. X. Wang, V. Neplokh, M. Tchernycheva, J. Nassar, P. Roca i Cabarrocas, Rusli, 2016, "Core-Shell Heterojunction Solar Cells Based on Disordered Silicon Nanowire Arrays", J. Phys. Chem. C, 120, 2962-2972, DOI: 10.1021/acs.jpcc.5b09618. Copyright (2016) American Chemical Society. Panel d is reproduced with permission from S. Misra, L. Yu, W. Chen, M. Foldyna, P. Roca i Cabarrocas, 2014, "A review on plasma-assisted VLS synthesis of silicon nanowires and radial junction solar cells", J. Phys. D: Appl. Phys. 47 393001-, DOI: 10.1021/acs.jpcc.5b09618. Copyright (2016) IOP Publishing.] 
As intuitive, this approach can be very useful also for axial structures where EBIC mapping and profiling can be used to optimize the growth parameters in order to achieve optimal junction properties. For instance, Otnes et al. ${ }^{26}$ used this approach to optimize the MOCVD growth of InP NWs containing an axial p-i-n junction for photovoltaic devices. In particular, the authors faced the challenge given by the n-type background doping of InP due to P-vacancies, which induced a fairly abrupt $p-n^{-}-n$ junction instead of the desired $p-i-n$ architecture, as pointed out by the red EBIC curve in Figure 8f. By using a dimethyl-zinc (DMZn) flow, a compensating p-doping was added to the middle segment to restore its intrinsic behavior. The authors were able to obtain a wide space charge region, as necessary for photovoltaic devices and to define the optimal dopant flow to be used to overcome the problem (green and blue curves).

Beside the analysis of the intra-wire properties, EBIC mapping also can be used to assess the wire-to-wire homogeneity of the electrical properties. For this purpose, the measurement can be performed in a top view or in a cross-sectional configuration. Tchoulfian et al. ${ }^{4}$, for instance, acquired an EBIC map on a tilted array to prove the homogeneity of the GaN core/shell NWs (Figure 9a and b). Thanks to the cleavage of one NW row, they proved that the depleted region, which produces the EBIC current, is reasonably homogeneous in all the observed wires. Togonal and coauthors, instead, combined different measurement configurations in order to perform a more systematic analysis of the array homogeneity ${ }^{55}$. They analyzed Si/a-Si NW radial junction solar cells obtained by a combination of a top-down etching with subsequent a-Si shell overgrowth. The authors observed the presence of few nonactive Si wires (labeled as "dead wires" in Figure 9c) by cross-sectional EBIC measurements where a clear difference with the active wires could be observed. The impact of the "dead wires" on the overall activity of the array can be appreciated by the top view measurement (reported in the inset of Figure 9c).

A particularly interesting case is the analysis of non-vertical NWs. In this case, the EBIC map can provide a feedback on the phenomena occurring at the points of contact between NWs, which can potentially induce leakage paths and therefore an electrical loss. Misra et al. ${ }^{56}$ analyzed solar cells based on self-assembled Si/a-Si NWs grown by PECVD, which present a highly irregular morphology. EBIC mapping on these non-vertical NWs was performed to visualize eventual current loss at morphological defects (Figure 9d). Surprisingly, EBIC map revealed that the array is electrically robust in particular at the NW crossings (highlighted with yellow arrows) where no decrease in the collected current is observed.

\section{Cathodololuminescence}

Cathodoluminescence (CL) is a spectroscopic technique which combines the analysis of the radiative recombination phenomena, typical of luminescence methods such as photoluminescence, and the spatial resolution of electron probe microscopy. With this regard, the main advantages of CL with respect to the photoluminescence are the ability of visualizing the morphology of the investigated sample and the nanoscale excitation which allows to achieve a very high resolution for the 
investigation of the optical properties. As discussed in Section 1, the spatial resolution depends on the interaction volume, the geometry of the sample, and the diffusion of the excited carriers (free carriers and/or excitons).

CL working principle can be schematized as in Figure 10a: the $\mathrm{e}^{-} / \mathrm{h}^{+}$pairs, locally excited by the electron beam, induce the emission of photons due to the radiative recombinations in the material. By associating the position of the scanning beam, with the SE image and the detected luminescence spectra in each point a socalled spectrum-image is acquired. In particular, it provides a 2D optical map of the investigated sample. We note that this is an excitation map. Indeed, while the excitation position is precisely defined, the exact recombination position is not known since the excited carriers (eventually bound by excitonic interaction) can move over a non-negligible distance. For instance, carrier diffusion towards a low-bandgap region results in a redshift in the photon emission ${ }^{57}$.

In order to extract from the spectrum-image useful information about the emitted luminescence, CL maps show the integrated intensity for each pixel. If the luminescence intensity is integrated over the whole detected spectrum, a spectrally integrated map (or panchromatic map) is obtained. Often the integration is done in a narrow wavelength range of interest to display the properties of a specific material or structure. In fact, the energy of the emitted photons reflects the local band structure which may depend on a large number of factors, such as the crystal structure, local composition and strain state ${ }^{58-60}$. An example is given in Figure $10 \mathrm{~b}$ where the SEM image of a GaN NW containing AlN/GaN multiple quantum discs and corresponding CL maps built by integrating the luminescence in different spectral ranges are shown. In this case, due to the strain arising from the heterostructure, photons with higher energy are emitted from the bottom part of the wire. This phenomenon cannot be observed in panchromatic maps since a spectral analysis of the emission is needed. For more details on this work, refer to ref. ${ }^{59}$.
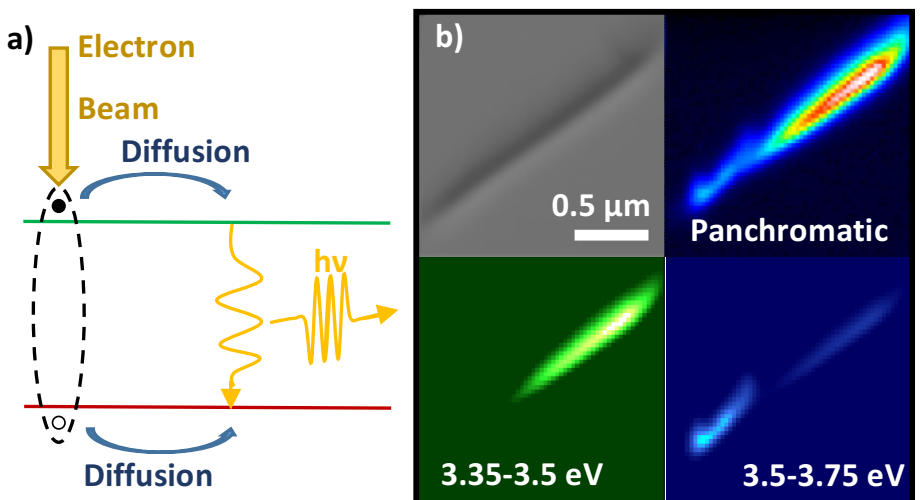

Figure 10 a) Schematic of the working principle of CL mapping of semiconductor materials; b) SEM image of a GaN NWs containing AlN/GaN MQDs and corresponding panchromatic, 3.35-3.5 eV and 3.5-3.75 eV filtered CL maps. 
The main application of CL mapping is the visualization of the optical transition energies and intensities ${ }^{61}$. Indeed, the variations of these quantities can be related to the presence of quantum confining heterostructures ${ }^{62-67}$ or to inhomogeneous composition and alloy fluctuations ${ }^{60,68,69}$. Another wide application of CL mapping is the assessment of material defects, which can be sites for both non-radiative and radiative recombination phenomena. In the first case, the presence of defects can be revealed by a local quenching of the CL emission ${ }^{70,71}$. The analysis of radiative defects, instead, relies on the knowledge of their emission wavelength, since specific types of defects are characterized by radiative recombination of carriers at determined energy levels. Indeed, point defects, stacking faults or dislocations create levels within the band gap which capture the charge carriers by allowing them to recombine at lower energies ${ }^{47,72,73}$.

In some cases (e.g. for materials containing closely packed nano-emitters ${ }^{62,63}$ ), even higher resolution is desirable. For this reasons, several variations of the CL setup have been developed in the last years ${ }^{61,62}$ : for instance, STEM-CL was demonstrated to be able to characterize the optical properties of closely-packed quantum $\operatorname{discs}^{62,63}$ or of 2D and 1D radiative and non-radiative defects with unmatched spatial resolution ${ }^{74,75}$.

Another important application of CL mapping, which has gained a strong interest in the recent years, is the analysis of the luminescence lifetime with nanometric spatial resolution, which can be applied to investigate the properties of quantum emitters or to assess the impact of localized defects ${ }^{57,76,77}$ (e.g. grain boundaries in solar cells ${ }^{78}$ ). This can be obtained by time-resolved CL measurements (TR-CL).

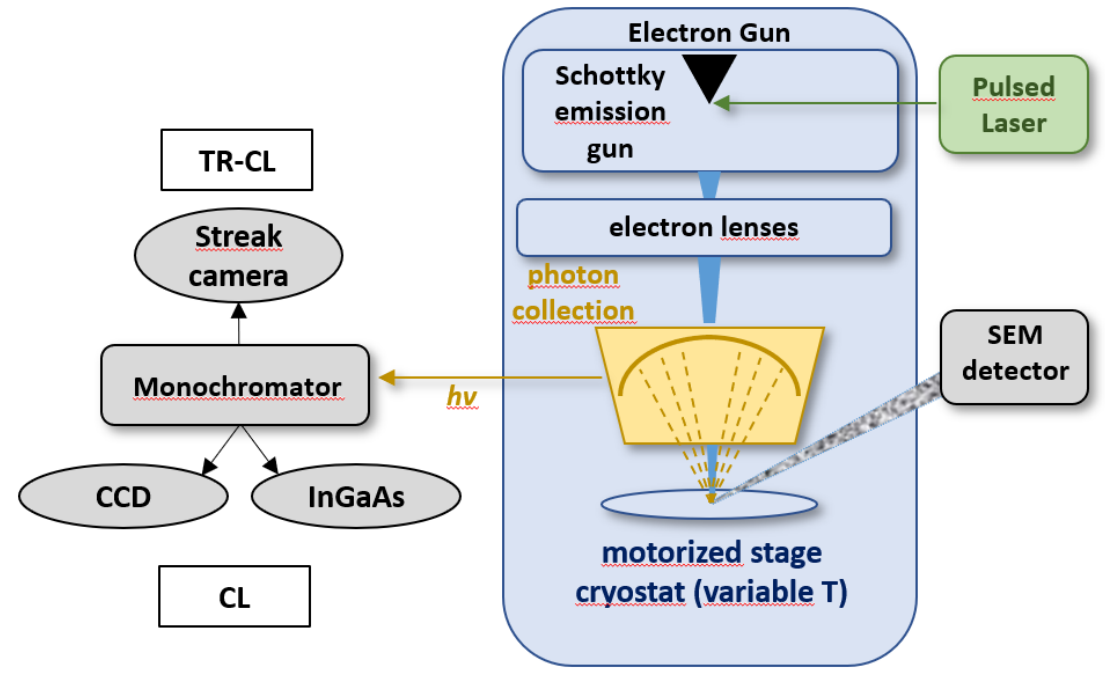

Figure 11 Simplified schematic of a SEM CL/TR-CL setup.

A schematic of a complete SEM CL/TR-CL tool equipped with time-resolved capabilities is shown in Figure 11. Different existing approaches providing temporal resolution of $\mathrm{CL}$ will be discussed in detail later in section 3.2. The electron probe 
is generated by the electron source (Schottky/Field-emission gun - FEG) and focused on the sample by means of a diaphragm characterized by the illumination aperture and by the magnetic lenses. The electron beam current injected into the sample is usually in the nA range. However, it can be tuned from a few tens of $\mathrm{pA}$ up to a few tens of nA, depending on the radiative efficiency and the high/low injection regime targeted. The CL emission generated in the far field by the probe/sample interaction (represented by the dashed yellow lines in Figure 11) is collected by a parabolic mirror, directed out of the microscope and analyzed by a monochromator and a detector array. To be noticed that using detectors made of different materials it is possible to be sensitive to different photon energy: for instance, a silicon CCD is used for visible and near-infrared wavelengths up to 1.1 $\mu \mathrm{m}$, while an InGaAs detector arrays can be used for wavelengths up to $1.7 \mu \mathrm{m}$. The stability of the sample stage is a key factor for high-resolution CL maps: the drift can reach values as low as $200 \mathrm{~nm}$ /hour, allowing high-resolution mapping of single NWs with low radiative efficiencies with total integration times up to several tens of minutes. In addition, CL setups usually allows low temperature measurements $(5 \mathrm{~K}-10 \mathrm{~K})$ thanks to a He cryostat, enabling the analysis of the optical properties as a function of the temperature.

\subsection{SEM-CL mapping applied to nanowires}

CL mapping is sensitive to the variation of the band gap in the investigated sample since the energy of the photons emitted upon a band-to-band transition changes; therefore, it can be used to assess the homogeneity of individual NWs. As already mentioned, one of the most common use of CL mapping is related to the analysis of the defects. In fact, the presence of defects can induce a quenching of the band edge transition (if non-radiative recombination phenomena occur in the defect) and/or the emission of less energetic photons, i.e. a red-shift of the emission (if radiative recombination phenomena occur in the defect). For instance, Bolinsson et al. investigated the variation of the optical properties of GaAs/AlGaAs core/shell NWs characterized by a high stacking fault density ${ }^{79}$. In this work, the authors proved that due to the high defect densities the NWs exhibit a luminescence peak below the material bandgap at $1.48 \mathrm{eV}$. In addition, the expected $1.52 \mathrm{eV}$ emission (zinc-blende GaAs) has been observed only in NWs containing large single crystal segments; in the other cases, the near band edge luminescence was strongly quenched (Figure 12a).

The possibility to have a high spatial resolution when mapping individual wires allows to study the origin of specific emissions. This advantage has several applications not only for the analysis of the defects, but in general for the investigation of elements which induce an intra-wire inhomogeneity, such as the crystal phase in materials which exhibit polytypism. Indeed, the luminescence emission is characteristic of the chemical nature of the material and its crystal phase. With this respect, an interesting study was carried out by Spirkoska et al. ${ }^{80}$ on GaAs NWs containing periods of wurzite (WZ) and zinc-blende (ZB) phases (Figure 12b). By scanning NWs dispersed on a substrate, the CL maps revealed optical emissions at different energies (by several tens of $\mathrm{meV}$ ) in the same nanostructures. The authors attribute 
this peculiar behavior to the presence of different crystal phases with nanometric dimensions and short diffusion lengths, which therefore act as a sequence of quantum heterostructures as schematically represented in Figure 12c. By the use of luminescence techniques, also the bandgap of these phases in NWs and their band alignment has been determined ${ }^{81}$.
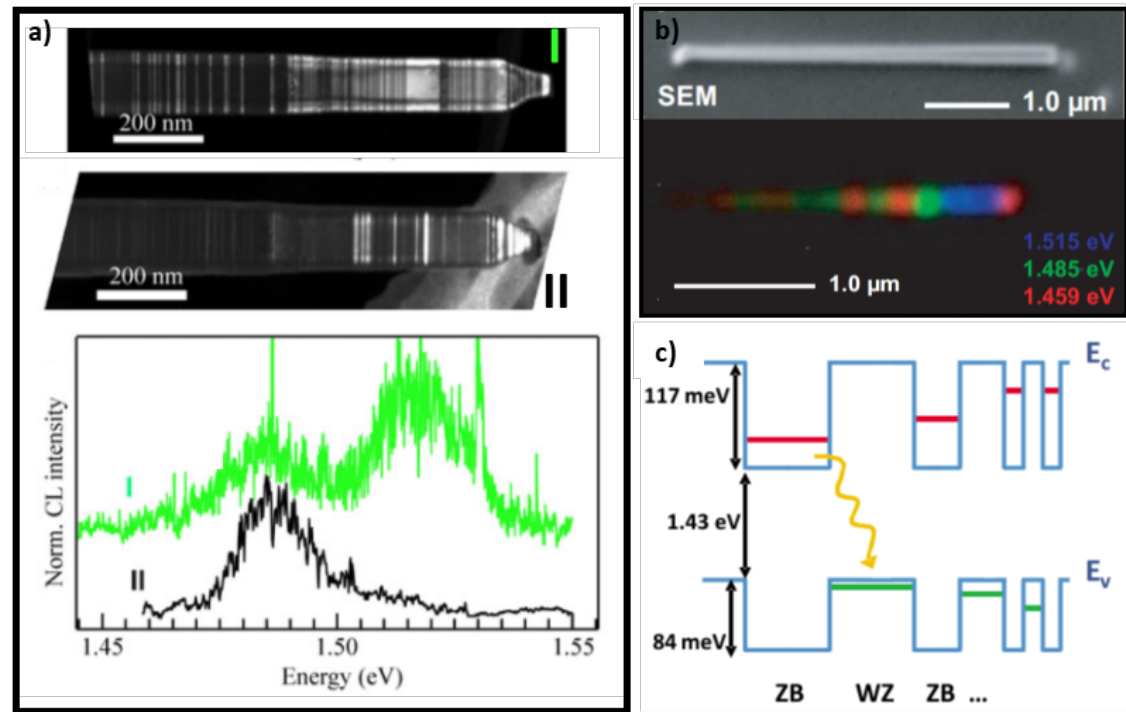

Figure 12 a) A combined CL and TEM study of two different single GaAs nanowires with a high stacking fault density and with/without a long zinc-blende GaAs segment (respectively labelled as "I" and "II"). In the CL spectra, the low energy emission of the defect (1.48 eV) is visible in both NWs, while the band edge transition is visible only in the NW I. b) SEM image and corresponding CL map of a single GaAs NW containing wurtzite and zinc-blende insertions (the color legend is displayed); c) Schematic of the band alignment of $Z B$ and $W Z$ insertions characterized by quantum confinement.

[Panel a is reproduced with permission from J. Bolinsson, M. Ek, J. Trägårdh, K. Mergenthaler, D. Jacobsson, M.-E. Pistol, L. Samuelson, A. Gustafsson, 2014, "GaAs/AlGaAs heterostructure nanowires studied by cathodoluminescence", Nano Res. 7(4), 473-490, DOI: 10.1007/s12274-014-0414-2. Copyright (2014) Springer-Nature. Panels b and c are reproduced with permission from D. Spirkoska, J. Arbiol, A. Gustafsson, S. Conesa-Boj, F. Glas, I. Zardo, M. Heigoldt, M. H. Gass, A. L. Bleloch, S. Estrade, M. Kaniber, J. Rossler, F. Peiro, J. R. Morante, G. Abstreiter, L. Samuelson, A. Fontcuberta i Morral, 2009, "Structural and optical properties of high quality zinc-blende/wurtzite GaAs nanowire heterostructures", Phys. Rev. B 80, 245325. Copyright (2009) American Physical Society]

In the last years, the ability to control the growth of nanometer-sized insertions in nanowires has taken advantage from the use of CL imaging for the analysis of quantum structures. For example, CL mapping enables the investigation of the optical properties of heterostructures containing quantum dots, wells and disks ${ }^{27,59,82}$. This research branch is particularly interesting for the development of new generation devices (single photons emitters and detectors, and other direction of quantum photonics). As an example, Heiss and coworkers studied GaAs/AlGaAs core/shell 
NWs where low Al ternary alloy quantum dots form with a self-assembly mechanism $^{83}$. The properties of these quantum dots are hardly predictable due to the complexity of the system which involves a core/shell structure, several ternary alloy compositions and different crystal phases. However, the peculiar composition of the dots allows their characterization by means of CL mapping. As shown in Figure $13 \mathrm{a}$, the optical map proves the presence of discontinuous structures within the wire exhibiting emission of photons at $\sim 1.8 \mathrm{eV}$, consistently with the expected energy levels of the quantum dots.

Figure 13 a) SEM image and corresponding CL map (filtered between 1.8 and $1.9 \mathrm{eV}$ ) of an individual GaAs/AlGaAs core/shell NW containing low Al quantum dots; b) SEM image of
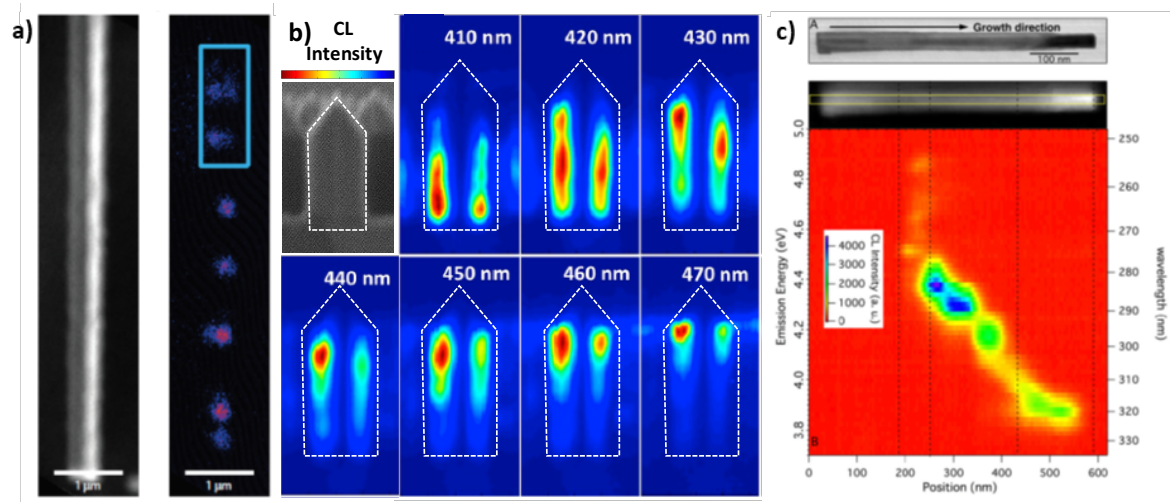

the inner cross-section of a GaN NWs containing radial In GaN insertions and corresponding CL maps at different emission wavelengths. The dashed white lines define the outer borders of the wire. CL relative intensity is represented by a color bar displayed in the inset. The maps show that the luminescence from the ternary alloy is redshjfted from the bottom to the top; c) SEM image, panchromatic CL map and spectrally resolved CL map of an individual AlGaN wire.

[Panel a is reproduced with permission M. Heiss, Y. Fontana, A. Gustafsson, G. Wüst, C. Magen, D. D. O'Regan, J.W. Luo, B. Ketterer, S. Conesa-Boj, A. V. Kuhlmann, J. Houel, E. Russo-Averchi, J. R. Morante, M. Cantoni, N. Marzari, J. Arbiol, A. Zunger, R. J.Warburton and A. Fontcuberta i Morral, 2013, "Self-assembled quantum dots in a nanowire system for quantum photonics", Nat. Mat. 12(5), 439-444, DOI: 10.1038/NMAT3557. Copyright (2012) Springer Nature. Panel b is reproduced with permission from M. Tchernycheva, V. Neplokh, H. Zhang, P. Lavenus, L. Rigutti, F. Bayle, F. H. Julien, A. Babichev, G. Jacopin, L. Largeau, R. Ciechonski, G. Vescovi, O. Kryliouk, 2015, "Coreshell InGaN/GaN nanowire light emitting diodes analyzed by electron beam induced current microscopy and cathodoluminescence mapping", Nanoscale 7, 11692-11701, DOI: 10.1039/C5NR00623F. Copyright (2012) The Royale Society of Chemistry. Panel c is reproduced with permission from A. Pierret, C. Bougerol, B. Gayral, M. Kociak, B. Daudin, 2013, "Probing alloy composition gradient and nanometer-scale carrier localization in single AlGaN nanowires by nanocathodoluminescence", Nanotech. 24, 305703, DOI: 10.1088/0957-4484/24/30/305703. Copyrigth (2013) IOP Publishing.]

The use of CL mapping has led also to the improvement of NW based LEDs and solar cells, where the optical properties can be finely tuned by inserting quantum structures. For instance, Tchernycheva et al. extensively investigated the fabrication of GaN NWs containing InGaN quantum wells for light emitting diode applications $3,27,30,84$. They found that one of the limiting factors of this technology was the spectrally broad electroluminescence of the final device. For this reason, the authors 
used CL mapping to probe the homogeneity of the composition of the ternary alloy along the length of single wires (Figure 13b) ${ }^{30}$. This analysis revealed an In gradient from the bottom (estimated around 10\%) to the top (estimated around 20\%).

Generally, the presence of alloy fluctuations is one of the most common limiting factors for optoelectronic devices, where a strict control over the optical properties is required and therefore a homogeneous composition is desirable. Indeed, a variation of the composition of III-V alloys induces a variation of the electronic band gap and, thus, of the optical emission ${ }^{68}$. For this reason, this phenomenon is largely studied by CL, especially in III-N NWs ${ }^{3,30}$. This the case of Pierret et al. ${ }^{60}$ who investigated the growth of AlGaN NWs for ultraviolet LEDs. In Figure 13c, the CL spectral map of a single NW is shown, indicating the energy (and the corresponding wavelength) of the photons emitted along the axis of the wire. The map shows that the emission wavelength shifts to higher values moving from the bottom to the top, indicating a Ga-poor composition on the bottom and a Ga-rich composition on the top. This analysis gave the possibility to investigate the growth regimes for the wires under observation. Moreover, CL allowed to investigate other growth-related phenomena such as the presence of alloy fluctuation in the order of 20-30 nm and the formation of a parasitic AlN shell around the wire ${ }^{85}$.

Due to the complex growth kinetics, the composition of III-V alloy NWs can be influenced by doping incorporation ${ }^{86,87}$. This is an important issue since many applications require doped ternary alloy materials (a common example is the use of InGaN for LEDs and InGaP and AlGaAs for tandem solar cells). In this scenario, the use of $\mathrm{CL}$ is fundamental for the design of nanowires containing electrical junctions. A representative example is given by the work of Bologna and coauthors ${ }^{86}$ who optimized the growth of InGaP NWs via template-assisted selective epitaxy (TASE). While a homogeneous composition was achieved in undoped wires, CL maps revealed a strong compositional variation in NWs containing an axial p-n junction (Figure 14a and b). In particular, they observed a blue-shift of the emission in a single NW containing a p-n junction (Figure 14c) associated to an increase of the Ga content in the p-doped segment, which is expected to have a negative effect on the spectral absorption of the light. To be noticed that the effect of the doping type on the alloy composition was already known for InGaP NWs grown by different methods ${ }^{87}$.

Beside the doping type, also the doping level can have an influence on the optical emission of III-V materials. This is commonly related to effects of band gap narrowing (leading to a red-shift of the luminescence wavelength) ${ }^{88,89}$ or to the increase of the optical band gap due to the population of some conductive states which increases the absorption edge of the material (Burstein-Moss shift) ${ }^{90}$. These shifts from the band edge transition can be locally observed and measured by CL analysis, which inspired Chen et al. ${ }^{91}$ to develop a CL map analysis method for the estimation of the doping level in single NWs (Figure 14d and e). In their work, the authors studied GaAs NWs with different doping levels and considered the energy peak shift and broadening as critical parameters. This approach paves the way to the use of CL as a non-destructive tool to estimate the doping level in individual nanowires, which is still a challenge in the development of NW based device. 
a)
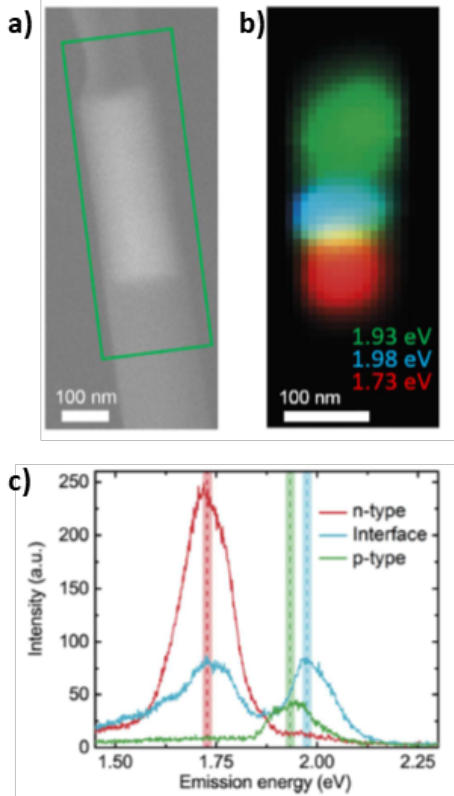

d)
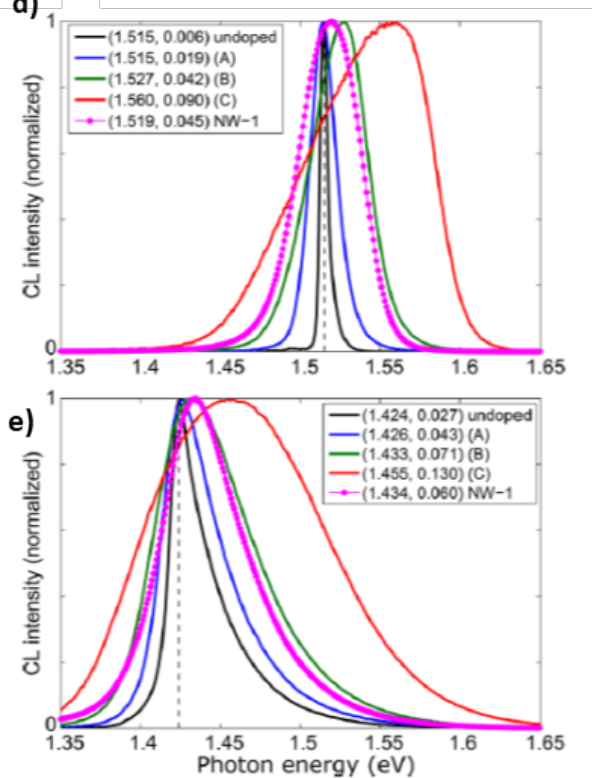

Figure 14 SEM image (a) and corresponding CL map (b) of an individual InGaP NW containing an axial p-n junction; c) CL spectra acquired along the axis of the NW from the $C L$ map in panel $b$ (the color legend is reported in panel b); $C L$ spectra acquired at low temperature (d) and at room temperature (e) of n-doped GaAs thin films with different doping levels compared to the spectra obtained from a single homogeneously doped GaAs NW.

[Panels a-c are reproduced with permission from N. Bologna, S. Wirths, L. Francaviglia, M. Campanini, H. Schmid, V. Theofylaktopoulos, K. E Moselund, A. Fontcuberta i Morral, R. Erni, H. Riel, M. D. Rossell, 2018, "Dopant-induced modifications of $\mathrm{Ga}_{x} \mathrm{In}_{(1-x)} \mathrm{P}$ nanowire based $\mathrm{p}-\mathrm{n}$ junctions monolithically integrated on Si (111)", ACS Applied Materials \& Interfaces. 10(38), 32588-32596, DOI: 10.1021/acsami.8b10770. Copyright (2012) American Chemical Society. Panels d and e are reproduced with permission from H.-C. Chen, C. Himwas, A. Scaccabarozzi, P. Rale, F.Oehler, A. Lemaitre, L. Lombez, J.-F. Guillemoles, M. Tchernycheva, J.-C. Harmand, A. Cattoni, S. Collin, 2017, "Determination of n-type doping level in single GaAs nanowires by cathodoluminescence. Nano Lett. 17, 66676675. DOI: 10.1021/acs.nanolett. 7b02620. Copyright (2017) American Chemical Society.]

\subsection{TR-CL analysis applied to nanowires}

While CL spectroscopy provides important and direct information on the optical properties of materials at the nanoscale, time-resolved CL (TR-CL) can be used to characterize the temporal evolution of the CL emission of a material after an excitation pulse. This technique measures the carrier dynamics at the nanoscale and can be used to determine material quality, carrier transfer or to identify CL emissions characterized by different carrier dynamics. To add temporal resolution to standard CL spectroscopy, three main techniques can be used and will be presented in the following sections.

(a) Beam-blanking techniques 
Historically, the first TR-CL experiments were performed using a beam blanking technique ${ }^{92-94}$. In such a configuration, the beam is deflected by applying a polarization to electrostatic deflection plates in the SEM column. In its standard configuration, the electron beam is swept out by applying a fast voltage pulse on the deflection plate. The temporal resolution of such a system is therefore limited by the rise time of the pulse generator, the spacing between the two deflection plates and the speed of the photodetector. Typically, such systems can achieve temporal resolutions of up to $100-200 \mathrm{ps}^{94,95}$. However, this technique can reach a temporal resolution up to $30 \mathrm{ps}$ when the beam blanker is used in conjugated mode in combination with two small apertures in the SEM column ${ }^{96}$. The main advantages of this technique are its relative ease of installation and its flexibility in pulse duration and repetition rate. On the other hand, this technique suffers from its relatively low temporal and spatial resolution since the electron beam is generally swept on the sample when it is deflected.
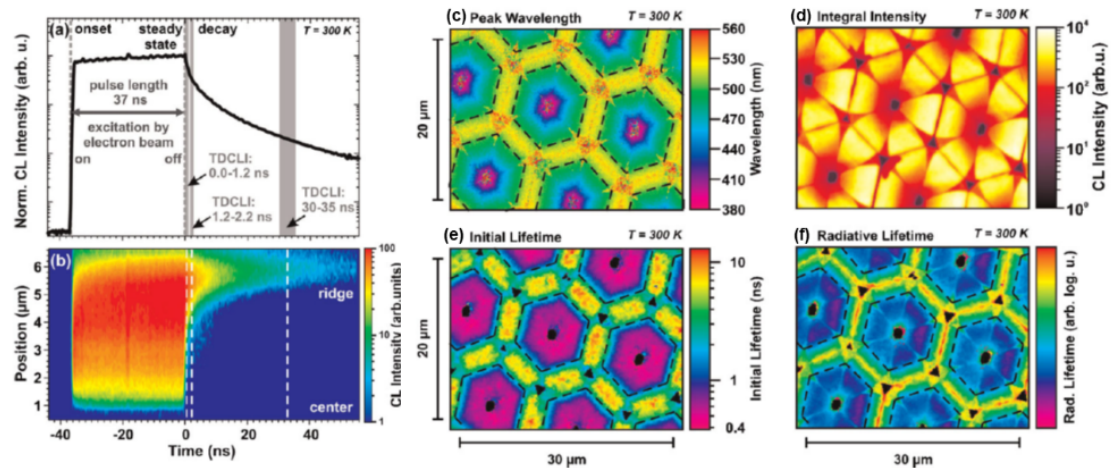

Figure 15 Transients recorded along a $\{1122\}$ facet of an inverted pyramid via time-resolved CL. a) Example of transient showing the establishment of steady state as well as the decay characteristics; b) Transient linescan over one facet from center to ridge; c) Mapping of the $C L$ peak wavelength from spectrally resolved measurements; d) Spectrally integrated intensity distribution gained from spectral mapping; e) Mapping of the initial lifetime of inverse pyramids evaluated from a mapping of local transients; $f$ ) Mapping of the qualitative radiative lifetime achieved by normalizing the initial lifetime distribution (e) to the steady state intensity distribution. The dashed hexagons mark the position of the $\mathrm{SiO}_{2}$ masking pads relative to the inverted pyramids.

[Panels a-f are reproduced with permission from S. Metzner, F. Bertram, C. Karbaum, T. Hempel, T. Wunderer, S. Schwaiger, F. Lipski, F. Scholz, C. Wächter, M. Jetter, P. Michler, J. Christen, 2011, "Spectrally and time-resolved cathodoluminescence microscopy of semipolar InGaN SQW on $(11 \overline{2} 2)$ and (1011) pyramid facets", PSSB. 248(3), 632-637, DOI: 10.1002/pssb.201046500. Copyright (2011) John Wiley and Sons.]

Such beam blanking TR-CL have often been used to characterize semiconductor nanostructures. In this respect, Metzner et al. carried out pioneering work in $2011^{97}$. They investigate the optical properties of $\mathrm{InGaN} / \mathrm{GaN}$ quantum wells grown on inverted pyramid surface thanks to spatially, spectrally, and time-resolved CL microscopy. By recording on the same spatial region the CL spectra and decay traces, the authors were able to determine for each pixel of the SEM image, the peak 
wavelength, integrated intensity, initial lifetime and radiative lifetime (Figure 15cf). This allowed them to correlate the emission properties (wavelength, lifetime) directly with the change in structural properties (indium content and quantum well thickness) from the center to the ridge of the inverted pyramids.

Since then, beam-blanking TR-CL has been successfully applied to other nanostructures. Fisher et al. correlated the structural, electronic, and optical properties of $\mathrm{AlN} / \mathrm{GaN}$ multiple quantum discs in GaN NWs grown on $\mathrm{Si}(111)$. In their study, the authors benefit from the use of TR-CL to determine the confinement regime in the heterostructure. In their experiments, they observed an increase of the CL lifetime when the temperature increases, which thus suggests that the quantum discs do not behave as quantum dots but rather as quantum wells ${ }^{98}$. As another example, Gačević et al. studied the influence of the composition, strain, and anisotropy of electric fields on the recombination dynamics of InGaN nanodisks in pencillike GaN NWs. Thanks to beam-blanking TR-CL experiments at low temperature, the authors observed the CL decay time at different positions of the NW to probe the local electric field. In particular, the fast recombination in the semipolar InGaN ( $\sim 0.5 \mathrm{~ns})$ revealed by TR-CL allowed them to demonstrate that the electric field across this section was low, which was associated with a near-unity electron-hole overlap $^{99}$.
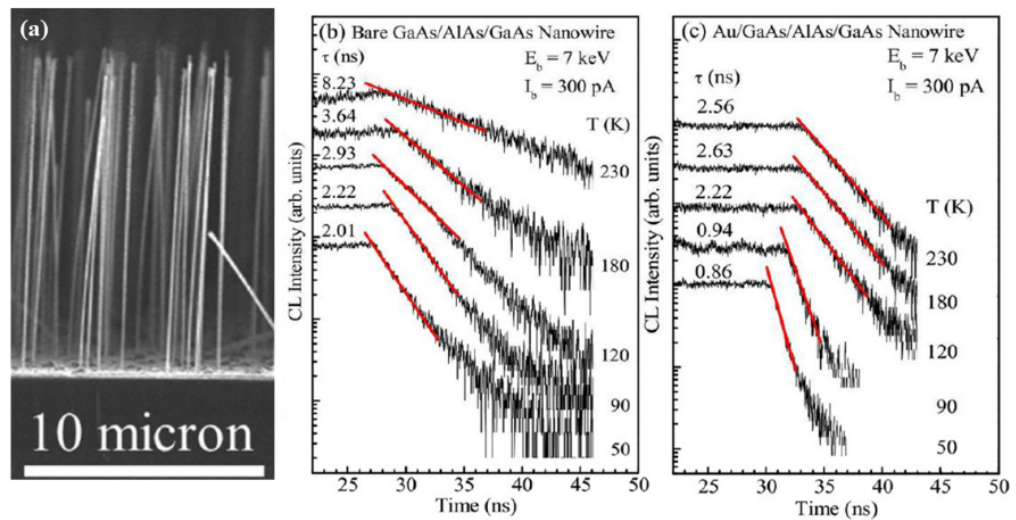

Figure 16 a) Side-view SEM image of the as-grown GaAs/AlAs/GaAs core-shell NWs. Local $C L$ decay transients for the bare (b) and Au-covered (c) NWs, respectively, at various temperatures from $50 \mathrm{~K}$ to $230 \mathrm{~K}$. Such a comparison reveals faster recombination when NWs are coating with gold suggesting an enhancement of radiative rate thanks to Purcell effect through exciton-plasmon coupling.

[Panels a-c are reproduced with permission from Y. Estrin, D. H. Rich, A. V. Kretinin , H. Shtrikman, 2013, "Influence of Metal Deposition on Exciton-Surface Plasmon Polariton Coupling in GaAs/AlAs/GaAs Core-Shell Nanowires Studied with Time-Resolved Cathodoluminescence", Nano Lett.13(4) 1602-1610 DOI: 10.1021/nl400015a. Copyright (2013) American Chemical Society.]

Beam-blanking TR-CL was also used to characterize the coupling of excitons to surface plasmon polaritons (SPPs) ${ }^{100}$. As an illustration, Estrin et al. characterized the influence of the deposited metal on the exciton-SPP coupling in GaAs/AlAs core-shell NWs (Figure 16a). By measuring the CL decay of bare NWs as function of temperature (Figure 16b) and comparing with the CL decays of Au- and Al- 
coated NWs (Figure 16c), the authors were able to deduce the Purcell enhancement factor that describes the improvement in the radiative recombination rate through the coupling of exciton to SPP. In particular, they showed that Au-coated NWs may have a Purcell enhancement factor of more than two while Al-coated NWs do not exhibit significant changes.

Finally, Donatini et al. recently used beam-blanking TR-CL to quantify the influence of the electron beam dose on excitonic diffusion in $\mathrm{ZnO} \mathrm{NWs}^{95}$. Using the TR-CL, the authors measured the CL decay time for different exposures. From these measurements, they deduced the surface recombination velocity as well as the exciton diffusion length and their dependence on the electron irradiation.

\section{(b) Laser driven electron beam}

In order to avoid the limitation of the electronics of the beam-blanking unit and to achieve a temporal resolution of a few picoseconds, a team led by J.-D. Ganière at EPFL developed in 2005 an optically driven electron gun ${ }^{101}$. This electron gun consists of a 20-nm-thick gold photocathode deposited on a quartz plate. By illuminating the photocathode with a $200 \mathrm{fs}$ pulsed laser emitting at $266 \mathrm{~nm}$, the photoelectric effect converts the photon pulse into an electron pulse. Thanks to this technique, the temporal resolution is limited only by the speed of the photodetector, which can be less than two picoseconds if a streak camera is used. For this reason, this technique is often referred to as picosecond TR-CL in the literature. Since then, other laser driven electron guns have been designed, to increase both the brightness and flexibility of use (Figure 17a) ${ }^{96}$.

Picosecond TR-CL was first applied to NWs in the investigation of the optical properties of $\mathrm{ZnO}$ NWs by Corfdir and coworkers ${ }^{102}$. In this paper, the authors benefit from the use of TR-CL to identify the biexcitonic emission in these nanostructures. The TR-CL technique helps them to confirm that excitons can be efficiently trapped by non-radiative defects closing basal stacking faults.

Moreover, picosecond TR-CL has also been used to directly observe the motion of excitons in $\mathrm{ZnO}$ microwires ${ }^{103}$. As described in Figure $17 \mathrm{~b}$, to put carriers in motion, the $\mathrm{ZnO}$ wires were bent. This bending creates a strain gradient along the cross section of the $\mathrm{ZnO}$ wire (Figure 17c). Then, thanks to the pulsed electron beam, excitons were created on the high energy side (inner region of the bent wire) and their motion has been monitored directly on the streak camera detector (Figure 17d). This motion is characterized by the shift in energy with time delay. Indeed, by converting the change in energy into a change in position along the strain gradient, the authors derived an exciton velocity of about $7 \mathrm{~nm} / \mathrm{ps}$, corresponding to an excitonic mobility of $1400 \pm 100 \mathrm{~cm}^{2} \mathrm{eV}^{-1} \mathrm{~s}^{-1}$. In the same vein, similar measurements were performed on an energy gradient in InGaN/GaN core-shell NWs, where the picosecond TR-CL helps to characterize the hopping process between localized states ${ }^{104}$. As a final example, by probing the carrier dynamics with a nanometer spatial and picosecond time resolution, picosecond TR-CL has been used to characterize the internal quantum efficiency of NW LEDs ${ }^{105}$. 

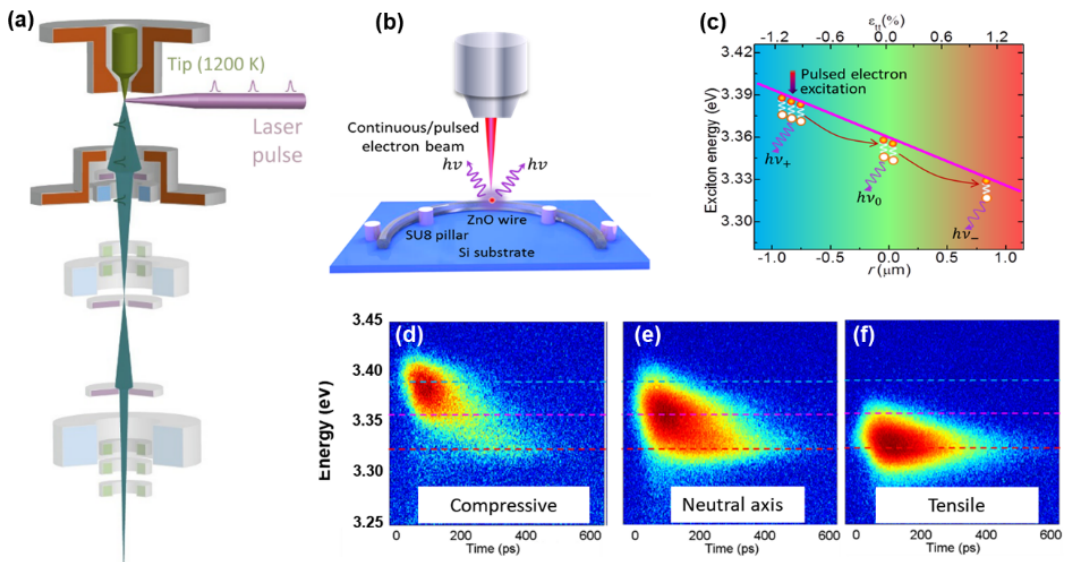

Figure 17 a) Schematics of the SEM column of a TR-CL setup in the pulsed-laser driven configuration.. (b) Schematic diagram of TR-CL measurements on the bending region of a bent $\mathrm{ZnO}$ microwire in a four point bending configuration. (c) Schematic diagram of exciton drift in a cross section of the bent $\mathrm{ZnO}$ microwire after pulsed electron excitation at the inner side (high-energy region). (d-f) Streak-camera images showing emission energy as a function of bent section $(d, e, f)$. The maps show a drift of exciton from compressive to tensile part, characterized by an overall redshift with time.

[Panel a is reproduced with permission from S.Meuret, , T.Coenen, E.Kieft, H.Zeijlemaker, M.Lätzel, S.Christiansen, S.Y.Woo, Y.H.Ra, Z.Mi, A.Polman, 2019, "Complementary cathodoluminescence lifetime imaging configurations in a scanning electron microscope", Ultramicroscopy

197, 28-38, DOI: 10.1016/j.ultramic.2018.11.006. Copyright (2019) Elsevier. Panels b-f are reproduced with permission from X. Fu, G. Jacopin, M. Shahmohammadi, R. Liu, M. Benameur, J.-D. Ganière, J. Feng, W. Guo, Z.-M. Liao, B. Deveaud, D. Yu, , 2014, "Exciton Drift in Semiconductors under Uniform Strain Gradients: Application to Bent ZnO Microwires", ACS Nano 8(4) 3412-3420 DOI: 10.1021/nn4062353. Copyright (2014) American Chemical Society.]

\section{(c) Hanbury-Brown and Twiss measurements}

More recently, a different technique has been developed to access to carrier dynamics at the nanoscale by taking advantage of the specificity of the excitation by an energetic electron beam.

Indeed, in the case of such excitation of a material, the electron-hole pair generation statistics differs from the one obtained with an optical stimulus. In the case of optical excitation, when a photon is absorbed into a material, it creates at best one electron-hole pair. In an electron microscope, the electron beam is very energetic (> $3 \mathrm{keV}$ ) and each incident electron can generate a large number of electron-hole pairs $(>1,000)$. Since these pairs are created simultaneously, there is a high probability that they will de-excite at the same time by emitting a photon. In such a case, this results in a strong bunching of photons. Moreover, by studying the autocorrelation function of the CL signal $\left(\mathrm{g}^{2}(\tau)\right)$ as a function of the delay, it is possible to characterize the carrier dynamics. Indeed, it has been shown that the $\mathrm{g}^{2}(\tau)$ evolves as the CL decay time ${ }^{106}$. 
In order to benefit from this unique property, Meuret and colleagues ${ }^{107}$ used a Hanbury Brown and Twiss intensity interferometer placed in the collection line to analyze the photon statistic of the CL emission (Figure 18a).

The main advantage of this technique is that it allows to access to carrier dynamics at the nanoscale without modifying the SEM column. Indeed, it only relies on the specificity of the unique electron-hole pair generation statistics by an electron beam and thus can be easily applied to any $\mathrm{SEM}^{96,107}$ or transmission electron microscope $(\mathrm{TEM})^{106,108}$.
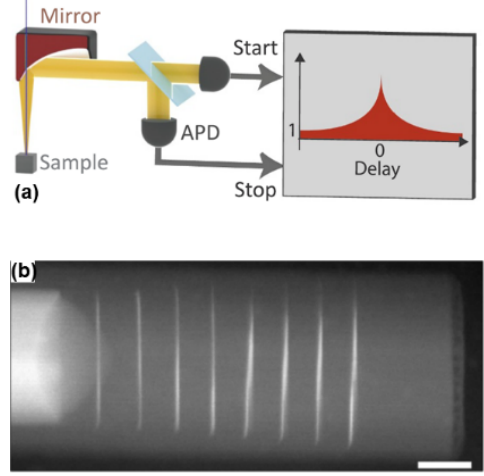
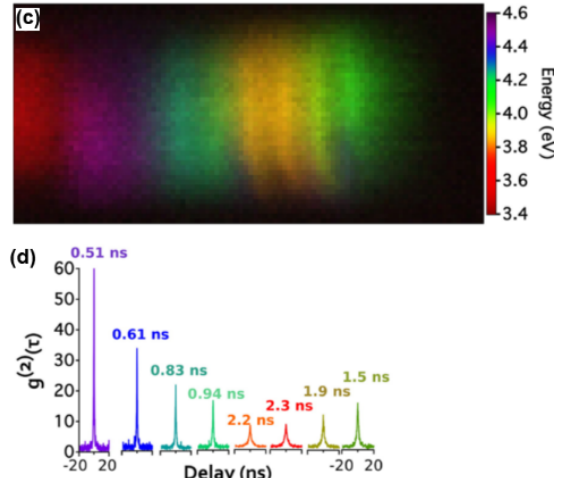

Figure 18 a) Schematic diagram of the CL set-up in autocorrelation mode where the light is collected by the parabolic mirror and sent to a Hanbury Brown and Twiss intensity interferometer. b) HAADF image of an AlN NW containing 8 GaN quantum discs with different thicknesses. Scale bar is $20 \mathrm{~nm}$; c) Compression of a spectral image acquired on an AlN NW containing $8 \mathrm{GaN}$ quantum discs and (d) series of $g^{2}(\tau)$ measured at each quantum disc position. These have been shifted on the abscissa to align with the corresponding quantum disc for clarity. The deduced lifetime is printed close to each bunching peak. The color change represents the evolution of emission wavelength for disc to disc.

[Panel a is reproduced with permission from S.Meuret, , T.Coenen, E.Kieft, H.Zeijlemaker, M.Lätzel, S.Christiansen, S.Y.Woo, Y.H.Ra, Z.Mi, A.Polman, 2019, "Complementary cathodoluminescence lifetime imaging configurations in a scanning electron microscope", Ultramicroscopy 197, 28-38, DOI: 10.1016/j.ultramic.2018.11.006. Copyright (2019) Elsevier. Panels b-d are reproduced with permission from Sophie Meuret, Luiz H. G. Tizei, Thomas Auzelle, Rudee Songmuang, Bruno Daudin, Bruno Gayral, Mathieu Kociak, 2016, "Lifetime Measurements Well below the Optical Diffraction Limit", ACS Photonics 3(7), 1157-1163, DOI: 10.1021/acsphotonics.6b00212. Copyright (2016) American Chemical Society.]

With this type of setup in a TEM, the lifetime of several quantum discs embedded in an AIN NW (Figure 18b) has been quantified with a spatial resolution well below the diffraction limit ${ }^{108}$. In these NWs, because of the growth conditions, the thickness is changing from disc to disc. Therefore, both the quantum confinement and the influence of the internal electric field is varying, leading to different emission energies for the eight quantum discs (Figure 18c). Due to the strong internal electric field in the GaN quantum discs, the change in thickness of a quantum disc induces a strong modification of the electron-hole overlap and therefore of the recombination probability and wavelength. By evaluating the $g^{2}(\tau)$ measured at each quantum disc position, the authors were able to correlate the quantum disc thickness 
and the CL emission energy to the radiative recombination rate. Using a similar configuration in a SEM, Meuret et al. used autocorrelation CL measurements to characterize the emission properties of GaN/InGaN quantum discs in $\mathrm{NWs}^{109}$.

\section{Conclusions}

Today the use of nanomaterials in electronic and optoelectronic applications witnesses a substantial growth. However, the complexity of their nanostructured three-dimensional morphology brings new conceptual and technological challenges. Understanding of the processes taking place at the nanoscale in individual nano-objects has become indispensable for their successful integration in functional devices. However, a reliable measurement of electrical and optical properties of nano-objects represents a big challenge. Electron beam induced current microscopy and cathodoluminescence can respond to this need. This is illustrated in the present chapter on the example of semiconductor nanowires. We have shown how these electron beam based techniques provide access to the key physical parameters, which govern the nanowire device efficiency, namely, to the material homogeneity, the bandgap, the radiative and non-radiative recombinations, the doping, the minority carrier diffusion lengths, etc. These parameters guide the optimization of the nanostructured devices and provide input for their advanced modeling.

\section{Acknowledgements}

This work has been partially financially supported by the French National Research Agency in the framework of the "Investissements d'avenir" program (ANR-15IDEX-02), by EU ERC project 'NanoHarvest' (grant no. 639052) and by H2020 ITN Marie Curie project 'INDEED’ (grant no. 722176).

\section{References}

${ }^{1}$ L. Reimer, Scanning Electron Microscopy: Physics of Image Formation and Microanalysis (Springer, 2013).

2 B.G. Yacobi and D.B. Holt, Cathodoluminescence Microscopy of Inorganic Solids (Springer Science \& Business Media, 2013).

3 M. Tchernycheva, P. Lavenus, H. Zhang, A.V. Babichev, G. Jacopin, M. Shahmohammadi, F.H. Julien, R. Ciechonski, G. Vescovi, and O. Kryliouk, Nano Lett. 14, 2456 (2014).

${ }^{4}$ P. Tchoulfian, F. Donatini, F. Levy, A. Dussaigne, P. Ferret, and J. Pernot, Nano Lett. 14, 3491 (2014).

${ }^{5}$ E.B. Yakimov, S.S. Borisov, and S.I. Zaitsev, Phys. Tech. Poluprovodiekov 41, 426 (2007). 
${ }^{6}$ P. Würfel, T. Trupke, T. Puzzer, E. Schäffer, W. Warta, and S.W. Glunz, J. Appl. Phys. 101, 123110 (2007).

${ }^{7}$ F.J. Schultes, T. Christian, R. Jones-Albertus, E. Pickett, K. Alberi, B. Fluegel, T. Liu, P. Misra, A. Sukiasyan, H. Yuen, and N.M. Haegel, Appl. Phys. Lett. 103, 242106 (2013).

${ }^{8}$ J. Wallentin, P. Wickert, M. Ek, A. Gustafsson, L. Reine Wallenberg, M.H. Magnusson, L. Samuelson, K. Deppert, and M.T. Borgström, Appl. Phys. Lett. 99, 253105 (2011).

${ }^{9}$ C.-C. Chang, C.-Y. Chi, M. Yao, N. Huang, C.-C. Chen, J. Theiss, A.W. Bushmaker, S. LaLumondiere, T.-W. Yeh, M.L. Povinelli, C. Zhou, P.D. Dapkus, and S.B. Cronin, Nano Lett. 12, 4484 (2012).

${ }^{10}$ Y. Arroyo Rojas Dasilva, R. Kozak, R. Erni, and M.D. Rossell, U1tramicroscopy 176, 11 (2017).

${ }^{11}$ C. Donolato, Phys. Status Solidi A 65, 649 (1981).

${ }^{12}$ C. Donolato, J. Appl. Phys. 54, 1314 (1983).

${ }^{13}$ C. Donolato, Appl. Phys. Lett. 43, 120 (1983).

${ }^{14}$ H.J. Leamy, J. Appl. Phys. 53, R51 (1982).

${ }^{15}$ L. Pasemann, Ultramicroscopy 6, 237 (1981).

${ }^{16}$ C.A. Klein, J. Appl. Phys. 39, 2029 (1968).

${ }^{17}$ C. Gutsche, R. Niepelt, M. Gnauck, A. Lysov, W. Prost, C. Ronning, and F.-J. Tegude, Nano Lett. 12, 1453 (2012).

${ }^{18}$ Z.Z. Bandić, P.M. Bridger, E.C. Piquette, and T.C. McGill, Appl. Phys. Lett. 72, 3166 (1998).

19 Z.Z. Bandić, P.M. Bridger, E.C. Piquette, and T.C. McGill, 8 $(2000)$.

${ }^{20}$ K.L. Bunker, Development and Application of Electron Beam Induced Current and Cathodoluminescence Analytical Techniques for Characterization of Gallium Nitride-Based Devices, North Carolina State University, 2004.

${ }^{21}$ D. Cavalcoli and A. Cavallini, Mater. Sci. Eng. 98 (1994).

${ }^{22}$ K. Durose, P.R. Edwards, and D.P. Halliday, J. Cryst. Growth 197, 733 (1999).

${ }^{23}$ I. Aberg, G. Vescovi, D. Asoli, U. Naseem, J.P. Gilboy, C. Sundvall, A. Dahlgren, K.E. Svensson, N. Anttu, M.T. Bjork, and L. Samuelson, IEEE J. Photovolt. 6, 185 (2016).

${ }^{24}$ W. Van Roosbroeck, J. Appl. Phys. 26, 380 (1955).

${ }^{25}$ D.E. Ioannou and C.A. Dimitriadis, IEEE Trans. Electron Devices 29, 445 (1982). 
${ }^{26}$ G. Otnes, E. Barrigón, C. Sundvall, K.E. Svensson, M. Heurlin, G. Siefer, L. Samuelson, I. Åberg, and M.T. Borgström, Nano Lett. 18, 3038 (2018).

${ }^{27}$ H. Zhang, G. Jacopin, V. Neplokh, L. Largeau, F.H. Julien, O. Kryliouk, and M. Tchernycheva, Nanotechnology 26, 465203 (2015).

28 V. Piazza, M. Vettori, A.A. Ahmed, P. Lavenus, F. Bayle, N. Chauvin, F.H. Julien, P. Regreny, G. Patriarche, A. Fave, M. Gendry, and M. Tchernycheva, Nanoscale 10, 20207 (2018).

${ }^{29}$ Ya.L. Shabel'nikova and E.B. Yakimov, J. Surf. Investig. X-Ray Synchrotron Neutron Tech. 6, 894 (2012).

${ }^{30}$ M. Tchernycheva, V. Neplokh, H. Zhang, P. Lavenus, L. Rigutti, F. Bayle, F.H. Julien, A. Babichev, G. Jacopin, L. Largeau, R. Ciechonski, G. Vescovi, and O. Kryliouk, Nanoscale 7, 11692 (2015). ${ }^{31}$ P. Lavenus, A. Messanvi, L. Rigutti, A. De Luna Bugallo, H. Zhang, F. Bayle, F.H. Julien, J. Eymery, C. Durand, and M. Tchernycheva, Nanotechnology 25, 255201 (2014).

${ }^{32}$ H. Zhang, X. Dai, N. Guan, A. Messanvi, V. Neplokh, V. Piazza, M. Vallo, C. Bougerol, F.H. Julien, and A. Babichev, ACS Appl. Mater. Interfaces 8, 26198 (2016).

${ }^{33}$ C.I. Bright, 50 Years Vac. Coat. Technol. Growth Soc. Vac. Coaters (2007).

${ }^{34}$ T.J. Coutts, T.O. Mason, J.D. Perkins, and D.S. Ginley, Proc Electrochem Soc 99, 274 (1999).

${ }^{35}$ O. Hultin, G. Otnes, M.T. Borgström, M. Björk, L. Samuelson, and K. Storm, Nano Lett. 16, 205 (2016).

${ }^{36}$ J. Alvarez, I. Ngo, M.-E. Gueunier-Farret, J.-P. Kleider, L. Yu, P.R. Cabarrocas, S. Perraud, E. Rouvière, C. Celle, and C. Mouchet, Nanoscale Res. Lett. 6, 110 (2011).

${ }^{37}$ J.-S. Hwang, M.-C. Kao, J.-M. Shiu, C.-N. Fan, S.-C. Ye, W.-S. Yu, H.-M. Lin, T.-Y. Lin, S. Chattopadhyay, L.-C. Chen, and K.-H. Chen, J. Phys. Chem. C 115, 21981 (2011).

${ }^{38}$ D. Mikulik, M. Ricci, G. Tutuncuoglu, F. Matteini, J. Vukajlovic, N. Vulic, E. Alarcon-Llado, and A. Fontcuberta i Morral, Nano Energy 41, 566 (2017).

${ }^{39}$ E. Halpern, G. Elias, A.V. Kretinin, H. Shtrikman, and Y. Rosenwaks, Appl. Phys. Lett. 100, 262105 (2012). 
${ }^{40}$ V. Piazza, S. Wirths, N. Bologna, A.A. Ahmed, F. Bayle, H. Schmid, F. Julien, and M. Tchernycheva, Appl. Phys. Lett. 114, 103101 (2019).

${ }^{41}$ P. Narchi, V. Neplokh, V. Piazza, T. Bearda, F. Bayle, M. Foldyna, C. Toccafondi, P. Prod'homme, M. Tchernycheva, and P.R. i Cabarrocas, Sol. Energy Mater. Sol. Cells 161, 263 (2017).

${ }^{42}$ I. Volotsenko, M. Molotskii, Z. Barkay, J. Marczewski, P. Grabiec, B. Jaroszewicz, G. Meshulam, E. Grunbaum, and Y. Rosenwaks, J. Appl. Phys. 107, 014510 (2010).

${ }^{43}$ L. Esaki, Phys. Rev. 109, 603 (1958).

${ }^{44}$ W. Guter and A.W. Bett, IEEE Trans. Electron Devices 53, 2216 (2006).

${ }^{45}$ X. Zeng, G. Otnes, M. Heurlin, R.T. Mourão, and M.T. Borgström, Nano Res. 11, 2523 (2018).

${ }^{46}$ H.C. Casey, B.I. Miller, and E. Pinkas, J. Appl. Phys. 44, 1281 (1973).

${ }^{47}$ Gilles Nogues, Thomas Auzelle, Martien Den Hertog, Bruno Gayral, and Bruno Daudin, Appl. Phys. Lett. 104, 102102 (2014).

${ }^{48}$ O. Demichel, M. Heiss, J. Bleuse, and H. Mariette, Appl Phys Lett 3 (n.d.).

${ }^{49}$ A. Solanki, P. Gentile, V. Calvo, G. Rosaz, B. Salem, V. Aimez, D. Drouin, and N. Pauc, Nano Energy 1, 714 (2012).

${ }^{50}$ D. Baek, J. Lee, and B. Choi, Mater. Res. Bull. 58, 157 (2014).

${ }^{51}$ L. Baird, C.P. Ong, R.A. Cole, N.M. Haegel, A.A. Talin, Q. Li, and G.T. Wang, Appl. Phys. Lett. 98, 132104 (2011).

${ }^{52}$ Y. Dan, K. Seo, K. Takei, J.H. Meza, A. Javey, and K.B. Crozier, Nano Lett. 11, 2527 (2011).

${ }^{53}$ M. Yang, D. Dvorak, K. Leistner, C. Damm, S.P. Watkins, and K.L. Kavanagh, Nanotechnology 30, 025701 (2019).

${ }^{54}$ S. Kono, T. Teraji, H. Kodama, and A. Sawabe, Diam. Relat. Mater. 63, 30 (2016).

${ }^{55}$ A.S. Togonal, M. Foldyna, W. Chen, J.X. Wang, V. Neplokh, M. Tchernycheva, J. Nassar, P. Roca i Cabarrocas, and Rusli, J. Phys. Chem. C 120, 2962 (2015).

${ }^{56}$ S. Misra, L. Yu, W. Chen, M. Foldyna, and P.R. i Cabarrocas, J. Phys. Appl. Phys. 47, 393001 (2014). 
${ }^{57}$ M. Merano, S. Sonderegger, A. Crottini, S. Collin, P. Renucci, E. Pelucchi, A. Malko, M.H. Baier, E. Kapon, B. Deveaud, and J.-D. Ganière, Nature 438, 479 (2005).

${ }^{58}$ M. Mattila, T. Hakkarainen, M. Mulot, and H. Lipsanen, Nanotechnology 17, 1580 (2006).

${ }^{59}$ V. Piazza, A. Babichev, L. Mancini, M. Morassi, P. Quach, F. Bayle, ludovic largeau, F.H. Julien, P. Rale, S. Collin, J.-C. Harmand, N. Gogneau, and M. Tchernycheva, Nanotechnology (2019).

${ }^{60}$ A. Pierret, C. Bougerol, B. Gayral, M. Kociak, and B. Daudin, Nanotechnology 24, 305703 (2013).

${ }^{61}$ M. Kociak and L.F. Zagonel, Ultramicroscopy 176, 112 (2017).

${ }^{62}$ L.F. Zagonel, S. Mazzucco, M. Tencé, K. March, R. Bernard, B. Laslier, G. Jacopin, M. Tchernycheva, L. Rigutti, F.H. Julien, R. Songmuang, and M. Kociak, Nano Lett. 11, 568 (2011).

${ }^{63}$ L.F. Zagonel, L. Rigutti, M. Tchernycheva, G. Jacopin, R. Songmuang, and M. Kociak, Nanotechnology 23, 455205 (2012).

${ }^{64}$ J.T. Griffiths, S. Zhang, B. Rouet-Leduc, W.Y. Fu, A. Bao, D. Zhu, D.J. Wallis, A. Howkins, I. Boyd, D. Stowe, M.J. Kappers, C.J. Humphreys, and R.A. Oliver, Nano Lett. (2015).

${ }^{65}$ L.H.G. Tizei, S. Meuret, K. March, K. Hestroffer, T. Auzelle, B. Daudin, and M. Kociak, Appl. Phys. Lett. 105, 143106 (2014).

${ }^{66}$ S.K. Lim, M. Brewster, F. Qian, Y. Li, C.M. Lieber, and S. Gradečak, Nano Lett. 9, 3940 (2009).

${ }^{67}$ G. Tourbot, C. Bougerol, F. Glas, L.F. Zagonel, Z. Mahfoud, S. Meuret, P. Gilet, M. Kociak, B. Gayral, and B. Daudin, Nanotechnology 23, 135703 (2012).

${ }^{68}$ K. Pantzas, G. Patriarche, D. Troadec, M. Kociak, N. Cherkashin, M. Hÿtch, J. Barjon, C. Tanguy, T. Rivera, S. Suresh, and A. Ougazzaden, J. Appl. Phys. 117, 055705 (2015).

${ }^{69}$ A. Pierret, C. Bougerol, M. den Hertog, B. Gayral, M. Kociak, H. Renevier, and B. Daudin, Phys. Status Solidi RRL - Rapid Res. Lett. 7, 868 (2013).

70 S.F. Chichibu, A. Uedono, T. Onuma, B.A. Haskell, A. Chakraborty, T. Koyama, P.T. Fini, S. Keller, S.P. DenBaars, J.S. Speck, U.K. Mishra, S. Nakamura, S. Yamaguchi, S. Kamiyama, H. Amano, I. Akasaki, J. Han, and T. Sota, Nat. Mater. 5, 810 (2006). 
${ }^{71}$ S.F. Chichibu, A. Uedono, T. Onuma, T. Sota, B.A. Haskell, S.P. DenBaars, J.S. Speck, and S. Nakamura, Appl. Phys. Lett. 86, 021914 (2005).

${ }^{72}$ H. Lei, H.S. Leipner, V. Bondarenko, and J. Schreiber, J. Phys. Condens. Matter 16, S279 (2004).

${ }^{73}$ R. Liu, A. Bell, F.A. Ponce, C.Q. Chen, J.W. Yang, and M.A. Khan, Appl. Phys. Lett. 86, 021908 (2005).

${ }^{74}$ M. Müller, G. Schmidt, S. Metzner, P. Veit, F. Bertram, S. Krylyuk, R. Debnath, J.-Y. Ha, B. Wen, P. Blanchard, A. Motayed, M.R. King, A.V. Davydov, and J. Christen, Jpn. J. Appl. Phys. 55, 05FF02 (2016).

${ }^{75}$ A. Urban, M. Müller, C. Karbaum, G. Schmidt, P. Veit, J. Malindretos, F. Bertram, J. Christen, and A. Rizzi, Nano Lett. 15, 5105 (2015).

${ }^{76}$ M. Grundmann, J. Christen, D. Bimberg, A. Fischer- Colbrie, and R. Hull, J. Appl. Phys. 66, 2214 (1989).

${ }^{77}$ X. Fu, G. Jacopin, M. Shahmohammadi, R. Liu, M. Benameur, J.D. Ganière, J. Feng, W. Guo, Z.-M. Liao, B. Deveaud, and D. Yu, ACS Nano 8, 3412 (2014).

${ }^{78}$ B.G. Mendis, D. Gachet, J.D. Major, and K. Durose, Phys. Rev. Lett. 115, (2015).

${ }^{79}$ J. Bolinsson, M. Ek, J. Trägårdh, K. Mergenthaler, D. Jacobsson, M.-E. Pistol, L. Samuelson, and A. Gustafsson, Nano Res. 7, 473 (2014).

${ }^{80}$ D. Spirkoska, J. Arbiol, A. Gustafsson, S. Conesa-Boj, F. Glas, I. Zardo, M. Heigoldt, M.H. Gass, A.L. Bleloch, S. Estrade, M. Kaniber, J. Rossler, F. Peiro, J.R. Morante, G. Abstreiter, L. Samuelson, and A. Fontcuberta i Morral, Phys. Rev. B 80, (2009).

${ }^{81}$ M. Heiss, S. Conesa-Boj, J. Ren, H.-H. Tseng, A. Gali, A. Rudolph, E. Uccelli, F. Peiró, J.R. Morante, D. Schuh, E. Reiger, E. Kaxiras, J. Arbiol, and A. Fontcuberta i Morral, Phys. Rev. B 83, (2011).

${ }^{82}$ B. Loitsch, M. Müller, J. Winnerl, P. Veit, D. Rudolph, G. Abstreiter, J.J. Finley, F. Bertram, J. Christen, and G. Koblmüller, New J. Phys. 18, 063009 (2016).

${ }^{83}$ M. Heiss, Y. Fontana, A. Gustafsson, G. Wüst, C. Magen, D.D. O'Regan, J.W. Luo, B. Ketterer, S. Conesa-Boj, A.V. Kuhlmann, J. Houel, E. Russo-Averchi, J.R. Morante, M. Cantoni, N. Marzari, J. 
Arbiol, A. Zunger, R.J. Warburton, and A. Fontcuberta i Morral, Nat. Mater. 12, 439 (2013).

${ }^{84}$ H. Zhang, N. Guan, V. Piazza, A. Kapoor, C. Bougerol, F.H. Julien, A.V. Babichev, N. Cavassilas, M. Bescond, F. Michelini, M. Foldyna, E. Gautier, C. Durand, J. Eymery, and M. Tchernycheva, J. Phys. Appl. Phys. 50, 484001 (2017).

${ }^{85}$ A. Pierret, C. Bougerol, S. Murcia-Mascaros, A. Cros, H. Renevier, B. Gayral, and B. Daudin, Nanotechnology 24, 115704 (2013).

${ }^{86}$ N. Bologna, S. Wirths, L. Francaviglia, M. Campanini, H. Schmid, V. Theofylaktopoulos, K.E. Moselund, A. Fontcuberta i Morral, R. Erni, H. Riel, and M.D. Rossell, ACS Appl. Mater. Interfaces 10, (2018).

${ }^{87}$ G. Otnes, M. Heurlin, X. Zeng, and M.T. Borgström, Nano Lett. 17, 702 (2017).

${ }^{88}$ R.A. Abram, G.N. Childs, and P.A. Saunderson, J. Phys. C Solid State Phys. 17, 6105 (1984).

${ }^{89}$ G. Borghs, K. Bhattacharyya, K. Deneffe, P. Van Mieghem, and R. Mertens, J. Appl. Phys. 66, 4381 (1989).

${ }^{90}$ D.M. Szmyd, P. Porro, A. Majerfeld, and S. Lagomarsino, J. Appl. Phys. 68, 2367 (1990).

${ }^{91}$ H.-L. Chen, C. Himwas, A. Scaccabarozzi, P. Rale, F. Oehler, A. Lemaitre, L. Lombez, J.-F. Guillemoles, M. Tchernycheva, J.-C. Harmand, A. Cattoni, and S. Collin, Nano Lett. 17, 6667 (2017).

${ }^{92}$ S.M. Davidson, J. Microsc. 110, 177 (1977).

${ }^{93}$ A. Steckenborn, J. Microsc. 118, 297 (1980).

${ }^{94}$ D. Bimberg, J. Christen, A. Steckenborn, G. Weimann, and W. Schlapp, J. Lumin. 30, 562 (1985).

${ }^{95}$ F. Donatini and J. Pernot, Nanotechnology 29, (2018).

${ }^{96}$ S. Meuret, M. Solà Garcia, T. Coenen, E. Kieft, H. Zeijlemaker, M. Lätzel, S. Christiansen, S.Y. Woo, Y.-H. Ra, Z. Mi, and A. Polman, Ultramicroscopy 197, 28 (2019).

${ }^{97}$ S. Metzner, F. Bertram, C. Karbaum, T. Hempel, T. Wunderer, S. Schwaiger, F. Lipski, F. Scholz, C. Wächter, M. Jetter, P. Michler, and J. Christen, Phys. Status Solidi B Basic Res. 248, 632 (2011).

${ }_{98}$ A.M. Fischer, K.W. Sun, F.A. Ponce, R. Songmuang, and E. Monroy, Appl. Phys. Express 5, 1 (2012).

${ }^{99}$ Gačević, N. Vukmirović, N. García-Lepetit, A. Torres-Pardo, M. Müller, S. Metzner, S. Albert, A. Bengoechea-Encabo, F. Bertram, P. 
Veit, J. Christen, J.M. González-Calbet, and E. Calleja, Phys. Rev. B 93, 1 (2016).

${ }^{100}$ Y. Estrin, D.H. Rich, A. V. Kretinin, and H. Shtrikman, Nano Lett. 13, 1602 (2013).

${ }^{101}$ M. Merano, S. Sonderegger, A. Crottini, S. Collin, P. Renucci, E. Pelucchi, A. Malko, M.H. Baier, E. Kapon, B. Deveaud, and J.-D. Ganière, Nature 438, 479 (2005).

${ }^{102}$ P. Corfdir, M. Abid, A. Mouti, P.A. Stadelmann, E. Papa, J.P. Ansermet, J.D. Ganiére, and B. Deveaud-Plédran, Nanotechnology 22, (2011).

${ }^{103}$ X. Fu, G. Jacopin, M. Shahmohammadi, R. Liu, M. Benameur, J.D. Ganière, J. Feng, W. Guo, Z.M. Liao, B. Deveaud, and D. Yu, ACS Nano 8, 3412 (2014).

${ }^{104}$ M. Shahmohammadi, J.D. Ganière, H. Zhang, R. Ciechonski, G. Vescovi, O. Kryliouk, M. Tchernycheva, and G. Jacopin, Nano Lett. 16, 243 (2016).

${ }^{105}$ W. Liu, C. Mounir, G. Rossbach, T. Schimpke, A. Avramescu, H.J. Lugauer, M. Strassburg, U. Schwarz, B. Deveaud, and G. Jacopin, Appl. Phys. Lett. 112, 052106 (2018).

106 S. Meuret, L.H.G. Tizei, T. Cazimajou, R. Bourrellier, H.C. Chang, F. Treussart, and M. Kociak, Phys. Rev. Lett. 114, 1 (2015).

${ }^{107}$ S. Meuret, T. Coenen, H. Zeijlemaker, M. Latzel, S. Christiansen, S. Conesa-Boj, and A. Polman, Phys. Rev. B 96, 035308 (2017).

${ }^{108}$ S. Meuret, L.H.G. Tizei, T. Auzelle, R. Songmuang, B. Daudin, B. Gayral, and M. Kociak, ACS Photonics 3, 1157 (2016).

${ }^{109}$ S. Meuret, T. Coenen, S.Y. Woo, Y.H. Ra, Z. Mi, and A. Polman, Nano Lett. 18, 2288 (2018). 